\title{
Stellar Population Properties and Evolution Analysis of NGC 628 with the Panchromatic Photometry
}

\author{
Hu Zou ${ }^{1,2}$, Wei Zhang ${ }^{1}$, Yanbin Yang ${ }^{1}$, Xu Zhou ${ }^{1}$, Zhaoji Jiang ${ }^{1}$, Jun Ma ${ }^{1}$, Zhenyu Wu ${ }^{1}$, \\ Jianghua $\mathrm{Wu}^{1}$, Tianmeng Zhang ${ }^{1}$, Zhou Fan ${ }^{1}$
}

\begin{abstract}
Panchromatic spectral energy distribution (SED) from the ultraviolet (UV), optical to infrared (IR) photometry of NGC 628, combined with the evolutionary stellar population synthesis, is used to derive the spatially resolved age, metallicity and reddening maps. These parameter distributions show that the bulge of this galaxy is a disk-like pseudobulge, which has the Sérsic index close to the exponential law, rich gas, and a young circumnuclear ring structure. We also discover the disk has two distinct regions with different radial age and metallicity gradients. The inner region is older and has a much steeper age gradient than the outer region of the disk. Both these two regions and the central young structure can be seen in the radial profile of the optical color. Based on the age and reddening distributions, we consider that the pseudobulge and disk are likely to have grown via the secular evolution, which is the redistribution of mass and energy through the angular momentum transport caused by the non-axisymmetric potential of the spirals. However, possible gas accretion events could affect the outer region of the disk, due to abundant Hi gas accumulating in the outer disk.
\end{abstract}

Subject headings: galaxies: evolution — galaxies: individual (NGC 628) — galaxies: photometry — galaxies: stellar content

\section{INTRODUCTION}

Galactic evolution at the early universe was dominated by the hierarchical clustering and merging that are violent and rapid, secular evolution will be most important in the far future, and now both processes might be equally fundamental (Kormendy \& Kennicutt

\footnotetext{
${ }^{1}$ National Astronomical Observatories, Chinese Academy of Sciences, Beijing 100012, China; zhouxu@bao.ac.cn

${ }^{2}$ Graduate University of Chinese Academy of Sciences, Beijing 100049, China
} 
2004). Recently, more and more observations have revealed that the bulge with the Sérsic index approximating the exponential law might be grown via the secular evolution of the disk (Kormendv 1993; Böker et al. 2002; Kormendy \& Kennicutt 2004; Fisher \& Drory 2008; Ganda et al. 2009; Fisher et al. 2009). This kind of bulge is called pseudobulge, which is very common in late-type galaxies (Böker et al. 2002). Pseudobulges have smaller Sérsic indices than that of the de Vaucouleurs (1948) law in classical bulges, central gas concentration, active star formation, nuclear rings, bars and/or spirals (Kormendy \& Kennicutt 2004). Non-axisymmetric gravitational potential by the bar, oval, and/or spiral structures rearranges the angular momentum and mass, makes gas infall, and triggers the central star formation to create a pseudobulge (Regan \& Teuben 2003; Kormendy \& Kennicutt 2004). The gas infall from the disk and the formation of the bulge are secular. In addition to the secular evolution, distant encounters, gravitational interactions in clusters, and gas accretions from the neighboring galaxies can affect the formation and evolution of the bulge and disk (Toomre \& Toomre 1972; Noguchi 1988; Cortés et al. 2006; Bournaud \& Combes 2002). Thus, isolated nearby galaxies, which have large apparent sizes and are far from the tidal interactions of other galaxies, are excellent objects to investigate the secular evolution of the disk and formation of the bulge.

Spatially resolved age, abundance and reddening distributions can help us to understand the galactic evolution. They provide some basic information about the star formation history, chemical compositions and evolution, and interstellar medium. Both Kong et al. (2000) and Li et al. (2004) adopted different evolutionary population synthesis (EPS) models to analyze the structure and evolution of a nearby Sab spiral galaxy (M81) using the photometric data of multiple intermediate-band filters from $3000 \AA$ and $10000 \AA$ in the Beijing-Arizona-TaiwanConnecticut (BATC) multicolor sky survey. EPS has become a very popular technique to study the properties of stellar populations in galaxies (Fioc \& Rocca-Volmerange 1997; Leitherer et al. 1999; Bruzual \& Charlot 2003; Kotulla et al. 2009) and it has been applied in many fields such as star clusters, galaxies, and galactic clusters (Abraham et al. 1996; Kong et al. 2000; Ma et al. 2009a).

In this paper, we will use the EPS model to derive the population properties (age, metallicity and intrinsic reddening) and gain the clues for the formation and evolution of a nearby face-on SA(s)c galaxy, NGC 628. This galaxy, also named M74, has an apparent size of about $10^{\prime}$ and it is isolated (Kamphuis \& Briggs 1992). Light decomposition and color distributions reveal that this galaxy has a disk-like bulge (i.e., pseudobulge) (Natali et al. 1992; Cornett et al. 1994; Sánchez et al. 2011; Ganda et al. 2009). Besides the fifteen intermediateband observations taken with the $60 / 90 \mathrm{~cm}$ Schmidt telescope of National Astronomical Observatories of China as also used by Kong et al. (2000) and Li et al. (2004), there are plenty of on-line archival data for this galaxy from various surveys and telescopes, such as the 
Galaxy Evolution Explorer (GALEX), Two Micron All Sky Survey (2MASS), and Spitzer space telescope. These panchromatic photometric data-set from UV to IR can considerably aid us to obtain more accurate estimations of the properties (age, metallicity and intrinsic reddening).

Previous studies on NGC 628 present various properties in respect of colors, stellar populations, abundance, and reddening, etc., which can be compared with our results. The color of the whole galaxy is very blue, ranging from the bluest of M33's colors to the bluest of M81's in UV - V (Cornett et al. 1994). Cornett et al. (1994) reported that the disk might have undergone significant star formations over the past 500 Myr by comparing the UV/optical colors of stellar population synthesis models with those of NGC 628. Natali et al. (1992) highlighted that two different stellar populations dominate the inner and outer disk of the galaxy. Sánchez et al. (2011) presented the age and abundance profiles calculated by the integral field spectroscopy in an unprecedented high spatial resolution. They found a young circumnuclear star formation region near the galactic center. Distinct components with different ages and metallicities can be easily discriminated in those profiles. Hodge (1976) and Kennicutt \& Hodge (1980) both used H $\alpha$ images to obtain several hundreds of His regions. Numerous HiI regions, tending to lie along the spiral arms, indicate that a number of stars have been forming recently in this nearby galaxy. Both Talent (1983) and Belley \& Roy (1992) discovered a notable radial metallicity gradient according to the spectrophotometry and narrow-band imaging of His regions. Reddening in Belley \& Roy (1992) does not display any trend as a function of the radial distance from the galactic center. Regan et al. (2006) investigated the $8 \mu \mathrm{m}$ emission from the polycyclic aromatic hydrocarbons (PAHs) for some disk galaxies (including NGC 628) and declared the $8 \mu \mathrm{m}$ PAH surface brightness could be used as an approximate tracer of the interstellar medium.

The paper is organized as follows. In Section 2, the observations from the BATC survey and the archival data are described. Detailed data reduction is given in Section 3 . The introduction of the EPS model and fitting method is provided in Section 4. Decomposition of the surface brightness profile and the distributions of age, metallicity and reddening as well as relevant comparisons are presented in Section 5. Discussions and conclusions are made in Section 6 and 7, respectively.

\section{OBSERVATIONS}

NGC 628, as one of the nearby galaxies in the BATC sky survey (Burstein et al. 1994), was observed by the $60 / 90 \mathrm{~cm}$ Schmidt telescope, which is deployed at the Xinglong Station belonging to National Astronomical Observatories of China. A $2048 \times 2048$ Ford Aerospace 
CCD with the pixel scale of $1.7^{\prime \prime}\left(15 \mu \mathrm{m}\right.$ pixel $\left.^{-1}\right)$ is mounted at the focal plane of the telescope (the focal ratio is $\mathrm{f} / 3$ ). The size of field of view (FOV) is about $58^{\prime} \times 58^{\prime}$. For the photometric system, 15 intermediate-band filters, covering the wavelength range of $3300-$ $10000 \AA$ with bandwidths of about $200-300 \AA$, are well designed to avoid strong emission lines of the sky light (Fan et al. 1996). The observation mode is divided into two parts: deep exposures for observations of the objects; short exposures at photometric nights for both the objects and standard stars to do flux calibrations (Zhou et al. 2001). The normal observations for all filters started in 1995 November and ended in 2004 February. Because of the bad image qualities of $a, b$, and $c$ filters, we reobserved NGC 628 in 2009 winter for these three bands. Table 1 summarizes the basic parameters of filters, the total exposure time for each band, and some information of calibrations.

Figure 1 displays the combined images of NGC 628 in three BATC bands with wavelengths centered on 3890,6660, and $9190 \AA$. The galaxy is a grand-designed spiral galaxy with two symmetric inner arms and multiple long and continuous outer arms (classified as Type 9 in the arm classes of Elmegreen \& Elmegreen (1987)). We can see in those figures that two clear spiral arms extend from the center to the disk. Some Hil regions can be clearly recognized near the spiral arms in the $6660 \AA$ band (this band covers the $\mathrm{H} \alpha$ emission line) and in the $3890 \AA$ (near the ultraviolet regions where massive young stellar populations generate strong emissions), while the stellar mass distribution of the galaxy is visibly reflected in the near-infrared wavelength of $9190 \AA$. The disk inclination is about $6^{\circ}$ (Shostak \& van der Kruit 1984; Kamphuis \& Briggs 1992) and the position angle of the major axis is about $25^{\circ}$. The redshift is about 0.0022 (Huchra et al. 1999) and the redshiftindependent distance is about $8.6 \mathrm{Mpc}$ (Herrmann et al. 2008). This distance together with the disc inclination and position angle is adopted throughout our paper.

In addition to the optical data of the BATC survey, the archival data of the ultraviolet and infrared observations are collected to obtained the panchromatic spectral energy distribution of the galaxy from the on-line database. In the Nearby Galaxy Survey (NGS) of GALEX, NGC 628 was imaged in the far-UV (FUV) and near-UV (NUV) bands, providing substantially accurate ultraviolet observations with photometric precision up to 0.05 and 0.03 mag in the FUV and NUV bands, respectively (Martin et al. 2005; Morrissey et al. 2007). Due to its bad fitting to the model of stellar population synthesis, whose spectral libraries lack extreme horizontal-branch stars in the old stellar populations as mentioned in Ma et al. (2009b), the GALEX FUV band is excluded in the subsequent analysis. XMM Optical Monitor (XMM OM) also observed this galaxy in three other ultraviolet bands (UVW1, UVM2, and UVW2) (Kuntz et al. 2008). The signal to noise ratio (SNR) of the UVW2 band is extremely low, so this band is not used in our study. The UVM2 band together with UVW1 reinforces the observation data in the near-UV region. Near-infrared observations of NGC 
628 in 2MASS $J, H$, and $K_{s}$ bands (Skrutskie et al. 2006) are gained. Since only a small core in the $K_{s}$ band (radius less than half an arcmin) has enough SNR and the public data of this band from the $2.3 \mathrm{~m}$ Bok telescope (McCarthy et al. 2001) is available, we replace the 2MASS $K_{s}$ observation by the $K_{s}$ image of the Bok telescope for a detailed description of the data reduction refer to Knapen et al. (2004)). Infrared observations in 3.6 and $4.5 \mu \mathrm{m}$ of the Infrared Array Camera (IRAC, see Fazio et al. (2004)) of the Spitzer space telescope (Werner et al. 2004) expand the infrared data of NGC 628. Other bands in this telescope, such as 5.8, 8.0, 24, 70, and $160 \mu \mathrm{m}$, are located in the radiation regions of gas and dust (Kennicutt et al. 2003). Our study puts emphasis on the star light emitted from the stellar content, so these bands are not used in the analysis of the stellar populations. The ultraviolet, optical and infrared information of those telescopes and surveys including the instruments, images, and corresponding references is listed in Table 2.

\section{Data Reduction}

\subsection{Processing Flow of BATC Images}

Subtraction of the bias and dark current and the flat-field correction were performed by the PIPLINE 1 procedure as described in Fan et al. (1996) once the CCD frames were observed. Astrometric information was appended to the image header during this step. Deep frames of each band were combined after shift and rotation to the same image center and orientation according to the star positions. Cosmic ray hits and bad pixel effects were fixed up during the image combination. Short exposures for both the object and the standard stars were taken at photometric nights. We use these images to perform the flux calibration for the above deep combined image as follows: 1) Four Oke-Gunn standard stars (Oke \& Gunn 1983) are extracted in short exposure images of the standard stars and their instrumental magnitudes are measured by a stellar photometric procedure (DAOPHOT; Stetson (1987)); 2) Comparing the instrumental magnitudes and calibrated $A B$ magnitudes of these standard stars, we get atmospheric extinction coefficient and instrument zeropoint; 3) Tens or hundreds of unsaturated bright stars are selected as secondary standard stars in a short exposure image of the object, which is observed under the same photometric condition as the standard stars; 4) according to the extinction coefficient and zeropoint, the instrumental magnitudes of these stars are converted to flux-calibrated AB magnitudes. 5) The average difference between the instrumental magnitudes of the same stars in the deep combined image and their AB magnitudes are calculated; 6) with this difference, an analog-to-digital unit (ADU) can be converted calibrated flux density unit of $\operatorname{ergs~s}^{-1} \mathrm{~cm}^{-2} \mathrm{~Hz}^{-1}$. Calibration error of each short exposure for the object contains two items: one is from the magnitude estimations of 
the standard stars and the other is from the differences of those secondary standard stars between instrumental magnitudes and AB ones. The total calibration error for each band as shown in the last column of Table 1 is the root mean square (RMS) of the calibration errors in all short exposures, the number of which is presented in the seventh column of Table 1.

\subsection{Background Subtraction and Image Scaling}

Sky backgrounds should be removed from the mosaics of all bands in order to acquire the intrinsic flux of the galaxy. For the GALEX mosaics, background images are provided, but the background near the center is overestimated if we check their sky background intensity images. The mosaics from the 2MASS, Bok $K_{s}$ band, and Spitzer were constructed after removal of the sky background by their own techniques (see references in Table 2). We obtain the smoothing sky background maps for all other mosaics by the polynomial fitting method, based on the remaining background pixels after masking the source signals extracted by SExtractor (Bertin \& Arnouts 1996) and the galaxy by circling it with temperate apertures for different bands. These fitted sky backgrounds are then removed from the original mosaics.

Due to the diverse pixel scales ranging from $0.75^{\prime \prime}$ to $1.7^{\prime \prime}$ and different directions of the mosaics, we adjust them to the same scale and direction as the BATC images (i.e., 1.7" per pixel, top of north and left of east). Take the Spitzer mosaic for example, the pixel size is $0.75^{\prime \prime}$ but that of the BATC mosaic is $1.7^{\prime \prime}$. We first calculate the equatorial coordinate for each pixel of the BATC image, and then find the corresponding pixel position in the Spitzer mosaic according to its astrometry. After counting how many Spitzer pixels (can be decimals) are covered by the area of $1.7 \times 1.7 \operatorname{arcsec}^{2}$ centered at this pixel position, we sum all the fluxes of those pixels and subsequently the adjustments of both the pixel scale and direction are completed simultaneously. During the adjustments, we transform the pixel values of all bands to $\mathrm{AB}$ top-of-atmosphere fluxes in unit of $10^{-30} \mathrm{ergs} \mathrm{s}^{-1} \mathrm{~cm}^{-2} \mathrm{~Hz}^{-1}$. For the Bok 2.2 $\mu \mathrm{m}$ image from the NASA/IPAC Extragalactic Database (NED), since its astrometry is not the same as ours and the magnitude zeropoint is uncertain, we do something special: several stars with astrometric positions in the BATC image are used to update the astrometry of this image; mosaic fluxes are recalibrated to the AB magnitude system by some selected bright star in the same field from the 2MASS catalogues.

Figure 2 displays the true color image of NGC 628, combined by three scaled mosaics of the NUV, narrow $\mathrm{H} \alpha$ (from NED), and $8 \mu \mathrm{m}$ bands, which are chosen to present the distributions of young stellar population and dust. From this image, we can find that the whole disk is very blue, implying much younger populations in its disk than in the bulge. Numerous Hir regions, identified as luminous spots, mostly lie along the spiral arms. Light in 
HiI regions comes from both the old galactic background stellar population and young stars, such as $\mathrm{O}$ and $\mathrm{B}$ type, which have been born recently as a simple stellar population. These regions are more complex than the galaxy itself and they will be specially treated in our series papers. So pixels belonging to 376 Hı regions in all mosaics are obliterated according to the catalogue of Fathi et al. (2007). We also find numerous filaments and substructures generated by the PAH emission in red color of Figure 2, indicating the gas/dust distribution across the disk in addition to the strong PAH radiation along the spiral arms.

\subsection{Image Smoothing and SED Extraction}

Mosaics are smoothed for reasons that: 1) different astrometric precisions for telescopes lead a single pixel to represent adjacent slightly different parts of the galaxy; 2) transformations and rotations induce position errors; 3) different observational conditions cause diverse image qualities (e.g., CCD noises and seeing); 4) SNR varies from the center to the edge of the galaxy so that the photometric accuracy decreases in low SNR areas. Smoothing can improve SNR but in return reduce the spatial resolution of the galaxy. We use the boxcar averaging method to smooth all the mosaics as expressed in the following formula:

$$
M_{I, J}=\frac{1}{w^{2}} \sum_{K=I-\frac{w}{2}}^{I+\frac{w}{2}} \sum_{L=J-\frac{w}{2}}^{J+\frac{w}{2}} F_{K, L},
$$

where $I$ and $J$ specify the pixel position in the image, $M_{I, J}$ is the smoothed flux of this pixel, $F_{K, L}$ is the original flux of any given pixel $(K, L)$, and $w$ is the smoothing window width (so called boxcar width), depending on the SNR of the specified pixel in the BATC $j$ band image. The $\operatorname{SNR}(R)$ of a pixel is defined as

$$
R=\frac{S}{N}=\frac{S}{\sqrt{S+\delta^{2}}},
$$

where $S$ is the pixel flux, $\mathrm{N}$ is the noise, and $\delta$ is the standard deviation of the global sky background. Here, SNR is similar to the nominal definition in a CCD image, and we only use it to calculate the boxcar width and estimate relative photometric errors for different bands. The window size for each pixel is determined by $w=2 \min \left\{\left[\frac{R_{m}}{2 R}\right], 5\right\}+1$ where $\left[\frac{R_{m}}{2 R}\right]$ is the minimum integer larger than $\frac{R_{m}}{2 R}$ and $R_{m}$ is set to 10 . The formula promises $w$ to be an odd number to obviate the smoothing bias due to asymmetry. It means that if SNR of the pixel is equal or larger than 10, the minimum smooth width is 3 pixels and if SNR is equal or less than 1 , the maximum width is 11 .

After smoothing the mosaics, we extract the observed SED for each pixel. The observed 
SED includes AB magnitudes of 23 bands from UV to IR, which are calculated as

$$
m^{\text {obs }}=-2.5 \log _{10} S-48.6,
$$

as long as the flux signal $S$ is larger than $3 \delta$. The magnitude error $(\sigma)$ is determined by

$$
\sigma=2.5 \log _{10}\left(\frac{S+N}{S}\right)=2.5 \log _{10}\left(1+\frac{1}{R}\right) .
$$

At least 15 out of 23 bands with valid magnitudes are required for each SED to fit with model ones as explained in the next section. As a result, we get 33242 SEDs and their errors for the whole galaxy (see Table 3 about the SEDs of 6 randomly sampled pixels).

\section{STELLAR POPULATION SYNTHESIS AND FITTING METHOD}

Evolutionary population synthesis has become a popular technique in studying different properties of stellar populations and the evolution histories of galaxies, since there were some significant progresses on the theory of stellar evolution (Renzini \& Voli 1981; Maeder \& Meynet 1989; Kippenhahn \& Weigert 1990; Schaller et al. 1992; Bertelli et al. 1994), especially, the diverse phases of evolution (Iben \& Renzini 1983; Schoenberner 1983; Vassiliadis \& Wood 1993; Groenewegen \& de Jong 1993) and the completeness of stellar observational and theoretical spectral libraries (Le Borgne et al. 2003; Pickles 1998; Leieune et al. 1997). Most fashionable synthesis models, like PEGASE (Fioc \& Rocca-Volmerange 1997), StarBurst99 (Leitherer et al. 1999), GALEXEV (Bruzual \& Charlot 2003), and GALEV (Kotulla et al. 2009), are used to study globular clusters (Ma et al. 2009a; Barmby et al. 2000), galaxies (Kong et al. 2000; Papovich et al. 2001; Li et al. 2004), and galactic clusters (Abraham et al. 1996), etc.

In the series papers on the study of nearby galaxies based on the photometric observations of the BATC multicolor sky survey program, Kong et al. (2000) first quantified the chemical abundance, age and reddening distributions of M81 using the synthesis models of SSP (from Bruzual A. \& Charlot (1993)). Li et al. (2004) then used those spectral energy distributions (13 intermediate band filters) to gain the age and metallicity distributions of M81 with the help of PEGASE, which contains exponentially decreasing star formation rates (SFRs). Comparison of the above two models are described detailedly in their papers. We intend to apply the PEGASE model to investigate the distributions of the stellar population properties in NGC 628. 


\subsection{PEGASE Model}

PEGASE is a spectrophotometric evolution model for starburst and evolved galaxies of the Hubble sequence attributed to the extension to the NIR atlas of synthetic spectra and revised stellar libraries including cold stellar parameters, stellar tracks of asymptotic giant branch (AGB), and post-AGB phase (Fioc \& Rocca-Volmerange 1997). The stellar tracks they adopt are mainly from the "Padova" group, which contain a wide range of chemical abundances, $Z=0.0001,0.0004,0.004,0.008,0.02,0.05$, and 0.1 with $Y=2.5 Z+0.23$ $\left(Z_{\odot}=0.02\right.$, where $Z$ is the metallicity abundance and $Y$ is the helium abundance). The initial stellar mass in the tracks ranges from $0.6 M_{\odot}$ to $120 M_{\odot}$, where in the track of $Z=$ 0.1 pseudo-tracks with mass larger than $9 M_{\odot}$ are generated from the corresponding masses in the $Z=0.02$ and $Z=0.05$ tracks.

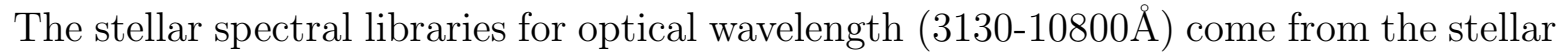
spectrophotometric atlas of Gunn \& Stryker (1983) which includes 175 stars with complete ranges of spectral type and luminosity class. For the far-UV (1230-3200A), stellar spectra are extracted from the International Ultraviolet Explorer (IUE) ESA/NASA libraries (Heck et al. 1984). In the extreme-UV (220-1230 А), spectra for the effective temper-

ate $T_{\text {eff }}<50000 \mathrm{~K}$ are calculated using the models of Kurucz (1992). The models of Clegg \& Middlemass (1987) are used at all wavelength for hotter stars $\left(T_{\text {eff }}>50000 \mathrm{~K}\right)$. For cold stars dominating the NIR $(1-5 \mu \mathrm{m})$ of the spectra, observational libraries when possible and synthetic spectra otherwise are adopted. Blackbody radiations supplement the NIR wavelength range of hotter stars. In the mid-infrared $(>5 \mu \mathrm{m})$ the analytic extension of Engelke (1992) is used for stars colder than $6000 \mathrm{~K}$.

Simple stellar population (SSP) is regarded as a simple system whose stars are born at the same time and with the same initial chemical compositions (typical example: globular clusters). SSPs can be calculated in PEGASE with initial mass functions (IMFs), stellar libraries, and stellar evolutionary tracks. PEGASE provides several commonly applied IMFs, and the Salpeter (1955) law with stellar mass larger than $0.1 M_{\odot}$ and less than $120 M_{\odot}$ is used to generate SSP models in this paper.

Galaxies are much more complex systems than globular clusters. Evolutionary composite stellar population (CSP) can more exactly describe the evolution history of galaxies. CSP is considered as a superimposition of SSPs of different ages. The integrated spectrum of CSP is synthesized with an IMF and a SFR for every metallicity. We assume each pixel of the mosaics to be such a CSP system that is composed of stars formed in different periods. There are three kinds of SFRs in PEGASE and the exponentially decreasing SFR with $\tau=15 \mathrm{Gyr}$ is used in our paper. Here, $\tau=15 \mathrm{Gyr}$ is typical for Sc galaxies (Bolzonella et al. 2000). 
Another feature of PEGASE is that the model takes into account the nebular emission (continuum and lines) generated by the ionized gas in the star-forming regions. Nevertheless, the regimes used to synthesize the spectral energy distribution for Hil regions are very outdated. We do not consider the nebular emission in the model spectra.

\subsection{Fitting Method}

For model spectra, both age from 0 to 20 Gyr and metallicity from 0.0001 to 0.1 are interpolated by the PEGASE code in the logarithmic space with steps of 0.01 . The Salpeter (1955) IMF and exponentially decreasing SFR are used to convolve these spectra to create CSPs of the specified metallicity. We use the dust extinction model of Cardelli et al. (1989) to redden the CSP spectra with a reddening step of 0.01 ranging from 0 to 1.0. By convolving the reddened CSP spectra with the filter transmission curves of all bands, we can obtain the model SEDs set in the ranges of different ages, abundances, and reddenings. The model SED contains convolved $\mathrm{AB}$ magnitudes of 23 bands computed as

$$
m_{i}^{\mathrm{csp}}=-2.5 \log _{10} \frac{\int_{\lambda} F_{\lambda}(t, Z, E) T_{i}(\lambda) d \lambda}{\int_{\lambda} T_{i}(\lambda) d \lambda}-48.6,
$$

where $i$ is the specified band index, $T_{i}$ is the corresponding filter transmission curve, $F(t, Z, E)$ is the CSP spectrum of the specified age $t$, metallicity $Z$ and reddening value $E$ in $E(B-V)$, and $m_{i}^{\text {csp }}$ is the resultant synthetical AB magnitude. By comparing the observed SED (see Section 3.3. with the synthetical model SEDs, we can simultaneously derive those three parameters $\left(t, Z\right.$, and $E$ ) for each pixel of NGC 628 via the $\chi^{2}$ minimum fitting method as described in Kong et al. (2000) and Ma et al. (2009b):

$$
\chi^{2}(t, Z, E)=\sum_{i=1}^{23} \frac{\left[m_{i}^{\mathrm{obs}}-m_{i}^{\mathrm{csp}}(t, Z, E)\right]^{2}}{\sigma_{i}^{2}},
$$

where $m_{i}^{\text {obs }}$ is the observed magnitude of the specified filter in the observed SED as shown in Equation 1, $\sigma_{i}$ is the observed magnitude errors as given in Equation 2, and $m_{i}^{\operatorname{csp}}(t, Z, E)$ is the corresponding synthetical magnitude in the model SED as shown Equation 3 .

The degenerate effect of age, metallicity, and dust reddening exists when we match the observed SEDs with the model ones predicted by EPS, since the ways of these three parameters to affecting spectra are similar. However, dust and gas absorb the UV radiation of star light and reradiate out in the infrared region. The multi-band photometric data from ultraviolet to infrared may aid in degrading this degeneracy. Before being fitted by the above method, the observed SEDs are corrected with the foreground reddening of the Galaxy by 
using the same reddening law of Cardelli et al. (1989). NGC 628 is located far away from the Galactic disk as the Galactic latitude of its center is $\mathrm{b}=-45.7^{\circ}$ (R.A.: $24.17^{\circ}$ and

Dec.: $\left.15.78^{\circ}\right)$. So the foreground Galactic reddening is small, about 0.07 mag in $E(B-V)$ (Schlegel et al. 1998).

\section{3. $\quad$ Fitting Results of Several Selected Pixels}

We randomly sample 6 pixels from different parts of NGC 628 to illustrate the SEDs, fitted parameters, and the fitting goodness: No. 1 near the galactic core, No. 2 from the inner region of the disk, No. 3 from the outer region of the disk, No. 4 from the inner spiral arm, No. 5 from the outer spiral arm, and No. 6 close to an Hil region (see Table 3). The positions of these six pixels are marked in the true color map of Figure 2 and the observed SEDs, matched model SEDs, and the corresponding model spectra are plotted in Figure 4. Excellent match to the model spectrum can be seen in this figure. We might coarsely conclude that: the core is oldest; age becomes younger distant from the center; spiral arms are younger than the rest components except for the places near the HII regions with much younger ages and richer abundances.

\section{Structure and Stellar Population Analysis of NGC 628}

Based on the spectral synthesis model and fitting method as described in the previous section, we obtain spatially resolved age, metallicity and intrinsic reddening distributions of NGC 628 after fitting the observed SED of each pixel with the models. In order to analyze the properties of different components, we first decompose the galaxy into two components in both the optical and near-infrared surface photometry. Then the stellar population analysis of the whole galaxy and its components is presented in the following sections.

\subsection{Bulge-disk Decomposition of the Surface Brightness Profiles}

As generally known in spiral galaxies, the bulge and disk exhibit many physical and dynamical differences. The bulge is usually brighter, older, and dynamically hotter than the disk. In order to analyze different components of NGC 628 with respect to the twodimensional features, we disassemble the galaxy into two main components. These two components are obtained by fitting the azimuthally averaged surface brightness with the exponential disk profile (Freeman 1970) and the Sérsic (1968) law following the iterative 
decomposition procedure of Kormendy (1977). In this decomposition method, two separate regions of the disk and bulge are chosen in the beginning. Then, a least square fitting of an exponential law to the radial surface brightness in the disk range is performed. The calculated exponential disk contribution is extrapolated to the bulge range and subtracted from the observed profile to get a first estimate of the bulge component, which has been fitted by the Sérsid (1968) law. In the same way, this fitted Sérsid (1968) law is extrapolated to the disk range and subtracted from the observed profile to get an estimate of the underlying disk, which be fitted by the exponential law again. The procedure repeats until all the parameters of two laws converge within a specified accuracy $\left(10^{-3}\right)$.

The radial profile is the corrected azimuthally-averaged values, given the disk inclination of $6^{\circ}$, the position angle of the major axis of $25^{\circ}$, and the distance of $8.6 \mathrm{Mpc}$ as previously mentioned. The value at each radius is defined as the average of the pixel values (here, surface brightness) within a suitably specified annulus of that radius centered on the galaxy nucleus. The profile error is calculated as the standard deviation of those pixel values. All radial profiles of different parameters (age, color, metallicity, and reddening) in the rest of the paper are computed in the same way with those adopted parameters (inclination, position angle and distance).

Left panel of Figure 3 shows the radial surface brightness profile of the BATC $d$ band (filled squares with error bars), whose effective wavelength is close to the broad $B$ band, and the decomposed bulge and disk components as shown in dashed curve and dotted line. We use the same regions where the bulge and disk clearly dominate the observed profile as adopted in the paper of Boroson (1981) to derive the decompositions iteratively: between $3.5^{\prime \prime}$ and $23.0^{\prime \prime}$ for the bulge and between $69.0^{\prime \prime}$ and $230.2^{\prime \prime}$ for the disk. The decomposition procedure gives the central surface brightness $\left(\mu_{0}^{B}\right)$ of $19.64 \pm 0.19 \mathrm{mag} \mathrm{arcsec}^{-2}$, the effective radius $\left(r_{e}\right)$ of $0.41 \pm 0.04 \mathrm{kpc}(0.16 \pm 0.02 \mathrm{arcmin})$, the Sérsic index $\left(n_{b}\right)$ of $0.82 \pm 0.08$ for the Sérsic law of the bulge, the disc central surface brightness $\mu_{0}^{D}=21.19 \pm 0.11 \mathrm{mag} \mathrm{arcsec}^{-2}$ and scale length $h=3.25 \pm 0.20 \mathrm{kpc}(1.30 \pm 0.08$ arcmin $)$ for the exponential law of the disk. We obtained a much smaller effective radius of the bulge than that of Boroson (1981), because the de Vaucouleurs (1948) law can not fit the bulge component very well when we check Figure 6 of their paper. Although large discrepancy for the bulge, the scale length of the disk approximate those of Boroson (1981) corresponding to their adopted distance of 12.2 Mpc. Actually, many exceptions when fitting the bulge with the de Vaucouleurs (1948) law were mentioned by McDonald et al. (2009) (and references therein), and it is more acceptable that the projected three-dimensional bulge profile is fitted by the generalized Sérsic (1968) law.

Although blue bands are used to derive the structural parameters of different compo- 
nents traditionally, their light profiles are substantially affected by the recent star formation and the extinction of the gas and dust. To the contrary, the NIR luminosity is a good tracer of the stellar mass and maps the distribution of the old stellar populations in galaxies. It is insensitive to the luminosity of young massive stars and unaffected by the extinction of the gas and dust for its ignorable extinction coefficient. Therefore, we also decompose the surface brightness profile of the NIR $K_{s}$ band into two components as shown in the right panel of Figure 3. The central surface brightness, the effective radius, and the Sérsic index of the bulge are $16.87 \pm 0.18 \mathrm{mag} \operatorname{arcsec}^{-2}, 0.52 \pm 0.04 \mathrm{kpc}(0.21 \pm 0.02 \mathrm{arcmin})$, and 1.31 \pm 0.11 , respectively. The disc central surface brightness and scale length are $19.35 \pm 0.10$ mag $\operatorname{arcsec}^{-2}$ and $2.51 \pm 0.12 \mathrm{kpc}(1.00 \pm 0.05$ arcmin), respectively. The effective radius, Sérsic index, and disc scale length are consistent with the structural parameters derived by Ganda et al. (2009) for the HST $H$ band photometry (Ganda et al. 2009), which give $r_{e}=0.21 \pm 0.002 \operatorname{arcmin}, n_{b}=1.23 \pm 0.01$, and $h=1.18 \pm 0.02$ arcmin. From the fitting parameters, we find that the disk profile decrease more dramatically in the NIR band than in blue optical band, since there are a number of young stellar populations located in the disk and they raise relatively more luminosity in the blue bands. The effective radius of the $K_{s}$ band is larger than that of the $d$ band, giving a more reliable span of the stellar mass of the bulge. We regard the region of $R<0.21^{\prime}$ as the region of the bulge, the region of $R>0.5^{\prime}$ where the profile starts to deviate from the exponential law as the region of the disk, and the region between these two areas as a transition zone.

\subsection{Age Distribution}

Figure 5 displays the age distributions of NGC 628 in 2D (left panel) and histogram (right panel). In the left panel of Figure 5, we find that age is oldest near the galactic core and becomes younger with the increasing radial distance (the age ranges from about 10 Gyr to 2.0 Gyr). Two apparent spiral arms in the disk, where considerable HiI regions are concentrated, are much younger $(\sim 1.3 \mathrm{Gyr})$ than any other components. They extend from the center to the outer region of this galaxy. The inter-arm areas are filled with relatively older stellar populations.

In order to derive the statistical properties of the stellar populations in more physical meaning, the near-infrared luminosity is used as the mass weight for each pixel in the galaxy image. Since the near-infrared luminosity is insensitive to young massive stars, and reddening in this wavelength range can be also ignored, the near-infrared bands, especially the $K_{s}$ band, are ideal tracers of the stellar mass (Cole et al. 2001; Kochanek et al. 2001). In the following of this paper, we will derive various statistical features (e.g., average values, histograms, and 
radial profiles) of age, metallicity, and reddening weighted by the $K_{s}$ band flux.

The average age of the whole galaxy, bulge and disk is about 4.9 Gyr, 7.5 Gyr and 4.4 Gyr, respectively. Half of the total stellar mass is younger than about 3.5 Gyr as seen in the right panel of Figure 5. In this figure, we can also see that there are quite a few extremely young stellar populations with age less than 1 Gyr. A majority of them are located close to the double spiral arms and some HiI regions as confirmed by their positions on the age map.

Sánchez et al. (2011) presented a wide-field IFS survey on NGC 628 and obtained 2D spectra in the FOV of about 6 arcmin in diameter with spatial resolution of about 2.7 arcsec. A dithering mode was adopted for the central pointing to increase the spatial resolution. They derived the global age of about 8.2 Gyr by both fitting the integrated spectrum of the whole observed galaxy to linearly combined SSPs and averaging the radial age distribution. The discrepancy of the average age with respect to our estimates mainly originates from the different size of the observed galactic area, which in our study is more than 2 times that of Sánchez et al. (2011). So considerable rather young stellar populations in the outer region of the disk are involved in the calculation of our average age.

\subsection{Two Distinct Disc Components}

The radial age profile in the left panel of Figure 6 shows that there are two distinct components in the disk, where we consider the radius range of $0.5-1.0^{\prime}$ as the inner region of the disk and the range from $1.0^{\prime}$ to $2.2^{\prime}$ as the outer region of the disk (separated at the radius of about $1.0^{\prime}$ ). This radial age profile is very similar to that of Sánchez et al. (2011) within the radius they analyzed regardless of the system uncertainties. The inner region of the disk is older and its age gradient is much steeper than that the outer region. The mean ages of these two components are about $7.2 \mathrm{Gyr}$ and $2.9 \mathrm{Gyr}$, respectively, and the slopes are $11.9 \pm 1.0 \mathrm{Gyr} \operatorname{arcmin}^{-1}\left(4.8 \mathrm{Gyr} \mathrm{kpc}^{-1}\right)$ for the inner region in the solid line and $1.2 \pm 0.1$

Gyr $\operatorname{arcmin}^{-1}\left(0.5 \mathrm{Gyr} \mathrm{kpc}^{-1}\right)$ for the outer region in the dashed line of the left panel of Figure 6.

Such distinct disc components in the age distribution should appear in the color profile which is related to the properties of the underlying stellar population, such as age and metallicity. We calculated the radial profile of the BATC $d-g$ color as plotted in the right panel of Figure 6. These two bands are selected according to their effective wavelengths approximating the broad bands of $B$ and $V$. The variation of this color profile is similar to that of the age profile: a flattening or even inverse tendency within $0.5^{\prime}$ and two different gradients in the region of $R>0.5^{\prime}$. 
Although the inner and outer regions are both in the exponential disk, the age of the inner region is much older than that of the outer one. The steeper age gradient of the inner region of the disk indicates this smaller component should experience a very long evolution history in an age span of about 7 Gyr. The outer region of the disk with much shallower gradient might be formed $2-3$ Gyr ago within a time interval of about 1 Gyr, which implies that NGC 628 might endure some encounter or accretion events and large number of gas fell in to produce new stars and generate the large-scale outer region of the disk in a very short time (see Section 6.3).

\subsection{Young Circumnuclear Ring Structure}

In the left panel of Figure 6, contrary to the disc components, an inverse age gradient resides in the bulge and the transition zone (also discovered in the $d-g$ color profile), while the oldest age up to 10 Gyr comes up at the galactocentric radius of about $0.5^{\prime}(1.3 \mathrm{kpc})$ instead of the galactic center. Relatively younger structure near the galactic core indicates that some processes like gas inflow directly through the galactic disk to the bulge might occur in the history and trigger the star formation. This may be also associated to the possible existence of a weak barred potential which induces the circumnuclear star formation as presented in the paper of Seigar (2002).

Natali et al. (1992) showed the broad band $U B V R I$ surface photometry of NGC 628, and found an obvious blue drop in their $B-V$ profile for $R<1.5 \mathrm{kpc}$, which can be predicted by models due to infall of gas in the bulge. Sub-mm CO (1-0) observation of Wakker \& Adler (1995) and infrared $2.3 \mu \mathrm{m}$ CO absorption spectroscopy of James \& Seigar (1999) displayed the existence of a circumnuclear star forming ring in the center of the galaxy. Cornett et al. (1994) used the UV surface photometry and discovered that the nuclear region has the overall morphological characteristics of spiral arm materials resembling those of M33 and might endure a significant star formation in the past few Gyrs predicted by the UV-optical colors.

In the paper of Sánchez et al. (2011), in addition to the decreasing age gradient in the inner region and possible shallow gradient in the outer region of the disk, an inverse age gradient was also obtained in a circumnuclear ring at about $25^{\prime \prime}$, which is very close to the position of our result. This kind of ring structure was also detected by Ganda et al. (2006) in their $\mathrm{H} \beta$ and $[\mathrm{O}$ III] distributions, obtained by the integral field spectroscopy with the SAURON Integral Field Unit pointing to the galactic core (about $33^{\prime \prime} \times 41^{\prime \prime}$ ). 


\subsection{Metallicity Distribution}

The metallicity $Z$, derived by SED fit, is converted to $[\mathrm{Fe} / \mathrm{H}]$ using the standard chemical composition of the Sun (Grevesse \& Sauval 1998). Related histogram and radial distribution are also calculated. In the left panel of Figure 7, we can see that the metallicity is lower near the core than in the outer regions and the abundance close to spiral arms is much richer than the inter-arms due to their poles of the frequently born place of massive stars.

Average $[\mathrm{Fe} / \mathrm{H}] \mathrm{s}$ of the whole galaxy, bulge, disk, and the inner and outer region of the disk are about $-0.77,-1.04,-0.78,-0.93$, and -0.71 dex, respectively. The derived global metallicity is lower than that of Sánchez et al. (2011), whose spectra mostly lie along the spiral arms and in the bulge. Much richer abundances of HiI regions in the spirals make the global metallicity of Sánchez et al. (2011) larger than ours. In the histogram of $[\mathrm{Fe} / \mathrm{H}]$ (middle panel of Figure 7), two components dominate this spiral galaxy: the older stellar population with poorer abundance and younger population with richer metallicity. The poor stellar population mostly lies within the galactic core and inter-arms, while the rich one mainly resides close to the spiral arms and the outer region of the disk. The distributions of these two populations take on a bimodal profile. We fit the overall distribution by a double Gaussian function which has two single Gaussian functions as its items. Parameters of these two Gaussian components are summarized in Table 4. The two components and the bimodal curve are respectively plotted in solid and dashed lines in the middle panel of Figure 7.

As presented by HiI observations of Talent (1983) and Belley \& Roy (1992), an obvious radial $\mathrm{O} / \mathrm{H}$ abundance gradient exists in the disk of NGC 628, which is 0.21 dex arcmin $^{-1}$ and $0.17 \pm 0.004$ dex $\operatorname{arcmin}^{-1}$, respectively corresponding to their adopted distances. Equally, a weak decreasing $[\mathrm{Fe} / \mathrm{H}]$ gradient located in the outer region of the disk is shown in the right panel of Figure 7. Sánchez et al. (2011) also obtained the radial stellar metallicity distribution, the profile of which is very similar to that of ours. The slope of this weak gradient is about $0.11 \pm 0.03 \mathrm{dex} \operatorname{arcmin}^{-1}\left(0.05 \mathrm{dex} \mathrm{kpc}^{-1}\right)$. Comparing those gradients with our result of 0.11 dex $\operatorname{arcmin}^{-1}$, we discover that the stellar metallicity gradient is somewhat lower than that of the gas abundance. It is notable that there is a reverse gradient of the inner region of the disk with a slope of $0.48 \pm 0.08 \mathrm{dex} \operatorname{arcmin}^{-1}\left(0.19 \mathrm{dex} \mathrm{kpc}{ }^{-1}\right)$. Such kind of abundance distribution in the disk provides fundamental constraints on the chemical evolution of NGC 628. 


\subsection{Intrinsic Reddening Distribution}

Both dust and gas absorb and scatter star light and make the galaxy redder. Extinctions are much larger near the UV bands than in the IR bands. Accurate photometry from the UV to infrared can derive reliable intrinsic reddening $(E(B-V))$ of NGC 628 as shown in the left panel of Figure 8. In this map, regions close to the spiral arms and the bulge hold relatively larger reddenings. The mean reddening of those regions close to spiral arms is about 0.38 and show no radial gradient, which is consistent with the observational measurements in Belley \& Roy (1992). Some inter-arm regions and parts of the outmost disk appears to be less reddened by dust and gas.

In order to confirm the reliability of the reddening value, dust emission of the Spitzer $8.0 \mu \mathrm{m}$ is compared with the reddening distribution. We construct the intensity map from the dust in Spitzer $8.0 \mu \mathrm{m}$ (right panel of Figure 8) dominated by the PAH emissions using

the method of Helou et al. (2004), who subtracted the background stellar fluxes from the observed fluxes. In the determination of the stellar fluxes in longer wavelengths than 3.6 $\mu \mathrm{m}$, Helou et al. (2004) extrapolated the $3.6 \mu \mathrm{m}$ flux using the stellar population model of StarBurst99 (Leitherer et al. 1999) to obtain the scale factor of 0.232 in $8.0 \mu \mathrm{m}$ (i.e., $D_{8.0}=I_{8.0}-0.232 I_{3.6}$, where $D_{8.0}$ is the intensity of the dust emission and $I_{3.6}$ and $I_{8.0}$ are the observed intensities in the Spitzer 3.6 and $8.0 \mu \mathrm{m}$, respectively). Superposed contours in the right map of Figure 8 show the distribution of $2.6 \mathrm{~mm} \mathrm{CO}$ (1-0) emission intensity from the Berkeley-Illinois-Maryland Association Survey of Nearby Galaxies (BIMA SONG) (Regan et al. 2001). Regan et al. (2006) concluded that the CO and $8 \mu \mathrm{m}$ emission morphologies are similar as also presented in this map and $8 \mu \mathrm{m}$ PAH surface brightness can be used as a possible tracer of the interstellar medium. Clearly strong PAH emissions preferentially distribute along the spiral arms where a number of HII regions radiate UV photons to photodissociate the PAHs around the molecule clouds and generate IR emissions. Compared with the reddening map, we discover that many of the regions with large reddening values in the galaxy show luminance peaks in the $8 \mu \mathrm{m}$ PAH image, implying the existence of abundant gas and dust. Some other regions with large reddenings might be caused by the absorption of dust lanes as checked in the optical observations with respect to the positions of primary dust lanes and reddening feathers, which were investigated by La Vigne et al. (2006).

Histogram in the left panel of Figure 9 shows that $E(B-V)$ varies from 0.1 to 0.6 and the mean reddening is about 0.31 . Average reddenings of the bulge, the disk, and the inner and outer regions of the disk are about $0.41,0.31,0.32$, and $0.31 \mathrm{mag}$, respectively. The radial distribution in the right panel of Figure 9 presents a very weak gradient of the intrinsic reddening with a slope of $0.015 \pm 0.002 \mathrm{mag} \operatorname{arcmin}^{-1}$. Several bumps in this plot 
are produced by the large reddening values near the spiral arms. We can see in the radial profile that the bulge as well as the transition zone has larger reddening values than the outside area, supporting the young circumnuclear ring structure holding abundant gas and dust. That is, a considerable volume of dust and gas should be deposited in the core of NGC 628, giving a rise of star formation in its recent history. In contrast, Kong et al. (2000) studied another nearby galaxy (M81) of Sab type using a similar method. Their results show that M81 has a much lower reddening range of $0.08-0.15 \mathrm{mag}$ in the bulge than ours and the disk reddening $(\sim 0.2 \mathrm{mag})$ is also somewhat smaller, implying that NGC 628 might be a gas-rich galaxy.

\section{Discussions}

\subsection{Pseudobulge and Secular Evolution}

The Sérsic indices in both optical and NIR bands as we have derived from the brightness profile fitting are close to 1 , which represents the exponential law. It has been increasingly discovered in recent years that bulges with the surface brightness profiles close to the exponential law are significantly different from those with larger Sérsic indices resembling the elliptical galaxies (Kormendv 1993; Kormendy \& Kennicutt 2004; Fisher \& Drory 2008; Ganda et al. 2009; Fisher et al. 2009). Now, two types of bulges are typically called: classical bulges that are dynamically hot and featureless and pseudobulges that retain the memory of the disk origin. The pseudobulges are very common in late-type galaxies $(75 \%$ of $77 \mathrm{Sd}-\mathrm{Sm}$ galaxies as given in Böker et al. (2002)). They have flatter shapes, kinematics dominated by rotation and corresponding smaller velocity dispersion, active star formation, nuclear bars, nuclear rings, and/or nuclear spirals and nearly exponential brightness profiles $\left(n_{b} \sim 1\right)$, while the classical bulges are dominated by random motions, contain old stellar populations, and are more similar to the E-type galaxies (Fisher \& Drorv 2008; Ganda et al. 2009; Fisher et al. 2009, and references therein). Specifically, Fisher et al. (2009) identified pseudobulges as those bulges containing nuclear bars, nuclear spirals, and/or nuclear rings and having the Sérsic index less than 2.

The Sérsic indices of NGC 628 are 0.83 for the blue band and 1.31 for the NIR band, both of which approximate the exponential law. Nuclear spiral arms are clearly seen in the UV band of this galaxy (Cornett et al. 1994). Moreover, the young ring structure detected in our age map and cental concentration of rich gas shown in the reddening map declare recent young star formation in the galactic center. Therefore, the bulge of NGC 628 belongs to that so-called disk-like pseudobulge. 
Unlike the classical bulges, which are typically merger-built, pseudobulges have the opportunity to be grown via the internal secular evolution of the disk (Kormendy \& Kennicutt 2004; Fisher et al. 2009). Disk galaxies can evolve by the rearrangement of mass and angular momentum driven by the non-axisymmetries of bars, ovals and/or spiral structures, which actuate the gas infall towards the galactic center, trigger the star formation, and build up the central mass concentrations of the pseudobulges (Kormendy \& Kennicutt 2004). In barred or oval disk galaxies, gravitational torques and/or shocks near their potential minimum cause the gas inflow and outflow, which can bring together the disk gas, hence trigger the star formation and make outer rings, inner rings, and central mass concentrations (pseudobulges) (Regan \& Teuben 2003; Fisher et al. 2009). In normal spiral galaxies without bars and ovals, spiral structures are maintained by the density wave that propagate through the disk. Shocks are formed as gas approaches and leaves the arms, making the gas lose the energy and sink into the center, and form the disk-like bulge (Kormendy \& Kennicutt 2004). NGC 628 is a late-type spiral galaxy without evident bars and ovals, so it is possible that its pseudobulge was formed by the secular evolution of the disk driven by the non-axisymmetric potential of spiral arms. Actually, Fisher et al. (2009) concluded that the median pseudobulges could have grown the current stellar mass at their present-day SFRs in 8 Gyr and their results are consistent with a scenario in which bulge growth via internal secular star formation. The age of the pseudobulge in NGC 628 as we obtained previously is about 7.5 Gyr, similar to the results of Fisher et al. (2009) and hence supporting the secular evolution.

Besides secular evolution, possible effects that build the pseudobulges include extremely gas-rich accretion events, distant gravitational encounters, and gravitational interactions in a cluster (Kormendy \& Kennicutt 2004; Fisher \& Drory 2008). NGC 628 is an isolated galaxies and no large galaxies are found within close distance which could generate possible tidal interactions (Kamphuis \& Briggs 1992). Thus, gravitational encounters and interactions should be unimportant for the formation of the pseudobulge in this galaxy. Hi observations by Kamphuis \& Briggs (1992) show two symmetrical high velocity complexes (HVCs) and an extended tail to the southwest. Gas accretion events from an external Hi-rich object may form the outer tail, and accretion can also create extreme velocity deviations making those HVCs visible. Kamphuis \& Briggs (1992) argued that we might be witnessing the flattening of the disk after accreting nearby companions long time ago according to the orbit of the tail, disc warp sustained by a steady gas infall, and complicated velocity distortion in the outer disk. However, it is extraordinarily uncertain whether this accretion-induced formation of pseudobulges can retain the disk-like properties. They also did not exclude the primordial origin that regards the observed gas distribution as the left-over of the initial formation of the galaxy. Nevertheless, the primordial origin and external accretion of gas disk in NGC 628 do not prevent this galaxy from forming the pseudobulge by the processes in the secular 
evolution and the secular evolution can still have an effect.

\subsection{Nucleus}

Both the age map (Figure 5) and the reddening map (Figure 8) show the nucleus with young age (about $3.5 \mathrm{Gyr}$ ) and rich gas (about $0.5 \mathrm{mag}$ in $E(B-V)$ ). Decomposition of the NIR $H$ band in extremely high resolution of the HST image also presents that the radial brightness profile exceeds the Sérsic law of the pseudobulge in the innermost of the galaxy (Ganda et al. 2009). This kind of compact nuclei hosts nuclear star clusters and they are very common in late-type galaxies (Böker et al. 2002). The nuclei are obviously distinguished from the ambient (pseudo)bulges and disk in the sense of a much smaller effective radius and higher effective surface brightness. Stellar populations of nuclei tend to have blue colors implying young age for the stars which contribute most of the light (typical example of M33 in Long et al. (2002)). The nucleus and the pseudobulge can both exist in the same galaxy but they show a great number of differences, making the nucleus harder to be understood. Maybe these tiny and dense nuclei were formed via dynamical friction driving the clusters sinking to the center as mentioned in Kormendy \& Kennicutt (2004). However, the origin and evolution of nuclei are quite complicated and its discussion is far beyond the ability with current results of our study.

\subsection{Scenarios of Disk Evolution}

In our results, we discover that the disk of NGC 628 can be divided into two parts: the inner region and the outer region. These two parts have different properties of stellar populations as far as age and chemical abundance be concerned. Disk dynamics points that the inner part of the disk shrinks and the outer part expands due to angular momentum transport caused by differential rotation and non-axisymmetric self-gravitating mode, such as spiral arms (Lynden-Bell 1979; Lin \& Pringle 1987; Tremaine 1989). This redistribution of mass and angular momentum results from the minimization of the total energy with the total angular momentum conservation. The gas contraction in the inner part of the disk could more easily trigger the star formation than in the outer part, and hence evolve more quickly. Merely by this kind of internal secular evolution, the disk can construct two different regions with a large age discrepancy. Certainly, one should confirm whether this process is fast enough to form the structures within the Hubble time. Nonaxisymmetry of the potential caused by the spiral arms can provide the engine for rapid

evolution as discussed in Kormendy \& Kennicutt (2004). On the other hand, Hi observa- 
tions of Shostak \& van der Kruit (1984) and Kamphuis \& Briggs (1992) display that most of the gas concentrates on the disk and an extensive tail lies to the southwest. Gas accretion events from an HI-rich object with $M_{\mathrm{HI}} \sim 9 \times 10^{8} M_{\odot}$ might occur long time ago and they affect only the outer disk loosely bounded by gravitation (Kamphuis \& Briggs 1992). Therefore, the accreted gas could rule the following star formation activities in the outer region, forming a low stellar-density and young outer region of the disk.

\section{CONCLUSION}

Recently, evolutionary stellar population synthesis models become more and more popular in studying the properties of the stellar populations in order to understand the star formation and evolution histories of galaxies. In this paper, we adopt one of the EPS models, PEGASE, to analyze the age, metallicity and reddening distributions of a nearby face-on spiral galaxy (NGC 628) based on multi-band photometric data ranging from UV to IR. Online archival data from GALEX, XMM OM, 2MASS, Bok telescope and Spitzer provide the ultraviolet and near-infrared photometry of this galaxy. The optical observations are obtained in the BATC survey with 15 intermediate bands ranging from $3000 \AA$ to about $1 \mu \mathrm{m}$. By comparing the photometric SED (totally 23 bands) with those of model ones calculated from the PEGASE synthetical spectra, we derive the properties of stellar population for each part of the galaxy (area of $1.7^{\prime \prime}$ in the BATC pixel size) and hence the spatially resolved age, metallicity and reddening maps.

Structure parameters are calculated in optical and NIR bands by fitting the surface brightness profiles with the Sérsid (1968) law for the bulge and exponential law for the disk, in order to discuss the properties of different components in NGC 628. The bulge size is 0.52 $\mathrm{kpc}$ and disc scale length is $2.51 \mathrm{kpc}$. The mean stellar age of the galaxy is about 4.9 Gyr, so young that a great number of stars might form in the recent history of $2-3$ Gyr. The age of the bulge is about $7.5 \mathrm{Gyr}$, presenting the oldest stellar population. The radial profile shows the age becomes younger and younger from the center to the outer disk. We confirm the presence of a young circumnuclear ring structure in the bulge and the transition zone within the galactocentric distance of about $0.5^{\prime}$. This young structure behaves to have flat and even inverse age gradient, showing active star formation. The disk can be divided into two parts as presented in the radial age profile: the old inner region and young outer region, which have distinct age gradients of $11.9 \mathrm{Gyr}_{\operatorname{arcmin}^{-1}}$ and $1.2 \mathrm{Gyr}_{\mathrm{arcmin}}{ }^{-1}$, respectively. The mean ages of these two regions are about 7.2 Gyr and 2.9 Gyr. Both the circumnuclear ring structure and two different regions of the disk can be clearly identified in the BATC $d-g$ color profile. 
Abundance map of NGC 628 in $[\mathrm{Fe} / \mathrm{H}]$ reveals that the bulge is consisted of old stellar populations with low metallicity $([\mathrm{Fe} / \mathrm{H}] \sim-1.04 \mathrm{dex})$ and the disk is relatively richer $([\mathrm{Fe} / \mathrm{H}]$ $\sim-0.78 \mathrm{dex})$. A very weak abundance gradient with slope of $0.11 \mathrm{dex} \operatorname{arcmin}^{-1}(0.05 \mathrm{dex}$ $\mathrm{kpc}^{-1}$ ) for the outer region of the disk is obtained in the radial profile, which is smaller than the gas abundance gradient obtained by the measurements of Hiı regions. Much larger [Fe/H] values in the metallicity map are discovered neighboring to HiI regions, where numerous young massive stars have been being formed. Reddening map shows obvious spiral-like lanes extending from the galactic core to the outer disk. Comparing with the IRAC $8 \mu \mathrm{m}$ dust emission image, we find that many parts of the reddening distribution correlate the PAH emissions. The global average reddening in $E(B-V)$ is about 0.31 . Reddening values of the bulge and disk are about 0.37 and 0.31 , respectively. A tiny gradient of the radial reddening distribution is found, whose slope is $0.015 \mathrm{mag} \operatorname{arcmin}^{-1}$.

At the end of this paper, the formation and evolution of different components of NGC 628 are discussed based on the results. As very common in late-type galaxies, the bulge of this galaxy is a pseudobulge, which has the Sérsic index close to the exponential law, young star formations and central spiral structures as seen in the UV band. The spiral structures generate the non-axisymmetric potential, make the gas fall into the center and form the central gas concentration which triggers the star formation and grows the pseudobulge. All these processes are secular and our results about the pseudobulge support its growth by the secular evolution. However, Hi gas distribution in the disk does not rule out the primordial origin and gas accretions affecting the formation of the pseudobulge. We also discover that a nucleus hosting star clusters can be obviously noticed, which has very young stellar populations and large reddening. Since NGC 628 is an isolated galaxy, gravitational interactions with other external galaxies are excluded in both the bulge and disk evolution. The secular evolution of the disk can form two distinct regions with a great age discrepancy, although gas accretion if existing, could also affect the outer region.

We thank the referee for his/her thoughtful comments and insightful suggestions that improve our paper greatly. This work is based in part on observations made with the Spitzer Space Telescope, which is operated by the Jet Propulsion Laboratory, California Institute of Technology under a contract with NASA. This publication makes use of data products from the Two Micron All Sky Survey, which is a joint project of the University of Massachusetts and the Infrared Processing and Analysis Center/California Institute of Technology, funded by the National Aeronautics and Space Administration and the National Science Foundation. We thank the SINGS (Spitzer), GALEX, and XMM-OM teams for making this research possible. We thank the observations of the $K_{s}$ band by the Bok telescope and CO emission by the BIMA SONG. This research has made use of the NASA/IPAC Extragalactic Database 
(NED) which is operated by the Jet Propulsion Laboratory, California Institute of Technology, under contract with the National Aeronautics and Space Administration. This work was supported by the Chinese National Natural Science Foundation grands No. 10873016, 10633020, 10603006, 10803007, 10903011, 11003021, and 11073032, and by National Basic Research Program of China (973 Program), No. 2007CB815403.

\section{REFERENCES}

Abraham, R. G., et al. 1996, ApJ, 471, 694

Barmby, P., Huchra, J. P., Brodie, J. P., Forbes, D. A., Schroder, L. L., \& Grillmair, C. J. 2000, AJ, 119, 727

Belley, J., \& Roy, J.-R. 1992, ApJS, 78, 61

Bertelli, G., Bressan, A., Chiosi, C., Fagotto, F., \& Nasi, E. 1994, A\&AS, 106, 275

Bertin, E., \& Arnouts, S. 1996, A\&AS, 117, 393

Böker, T., Laine, S., van der Marel, R. P., Sarzi, M., Rix, H.-W., Ho, L. C., \& Shields, J. C. 2002, AJ, 123, 1389

Bolzonella, M., Miralles, J.-M., \& Pelló, R. 2000, A\&A, 363, 476

Boroson, T. 1981, ApJS, 46, 177

Bournaud, F., \& Combes, F. 2002, A\&A, 392, 83

Bruzual, G., \& Charlot, S. 2003, MNRAS, 344, 1000

Bruzual A., G., \& Charlot, S. 1993, ApJ, 405, 538

Burstein, D., et al. 1994, Bulletin of the American Astronomical Society, 26, 1372

Cardelli, J. A., Clayton, G. C., \& Mathis, J. S. 1989, ApJ, 345, 245

Clegg, R. E. S., \& Middlemass, D. 1987, MNRAS, 228, 759

Cole, S., et al. 2001, MNRAS, 326, 255

Cornett, R. H., et al. 1994, ApJ, 426, 553

Cortés, J. R., Kenney, J. D. P., \& Hardy, E. 2006, AJ, 131, 747 
de Vaucouleurs, G. 1948, Annales d'Astrophysique, 11, 247

Elmegreen, D. M., \& Elmegreen, B. G. 1987, ApJ, 314, 3

Engelke, C. W. 1992, AJ, 104, 1248

Fan, X., et al. 1996, AJ, 112, 628

Fathi, K., Beckman, J. E., Zurita, A., Relaño, M., Knapen, J. H., Daigle, O., Hernandez, O., \& Carignan, C. 2007, A\&A, 466, 905

Fazio, G. G., et al. 2004, ApJS, 154, 10

Fioc, M., \& Rocca-Volmerange, B. 1997, A\&A, 326, 950

Fisher, D. B., \& Drory, N. 2008, AJ, 136, 773

Fisher, D. B., Drory, N., \& Fabricius, M. H. 2009, ApJ, 697, 630

Freeman, K. C. 1970, ApJ, 160, 811

Ganda, K., Falcón-Barroso, J., Peletier, R. F., Cappellari, M., Emsellem, E., McDermid, R. M., de Zeeuw, P. T., \& Carollo, C. M. 2006, MNRAS, 367, 46

Ganda, K., Peletier, R. F., Balcells, M., \& Falcón-Barroso, J. 2009, MNRAS, 395, 1669

Grevesse, N., \& Sauval, A. J. 1998, Space Science Reviews, 85, 161

Groenewegen, M. A. T., \& de Jong, T. 1993, A\&A, 267, 410

Gunn, J. E., \& Stryker, L. L. 1983, ApJS, 52, 121

Heck, A., Egret, D., Jaschek, M., \& Jaschek, C. 1984, A\&AS, 57, 213

Helou, G., et al. 2004, ApJS, 154, 253

Herrmann, K. A., Ciardullo, R., Feldmeier, J. J., \& Vinciguerra, M. 2008, ApJ, 683, 630

Hodge, P. W. 1976, ApJ, 205, 728

Huchra, J. P., Vogeley, M. S., \& Geller, M. J. 1999, ApJS, 121, 287

Iben, I., Jr., \& Renzini, A. 1983, ARA\&A, 21, 271

James, P. A., \& Seigar, M. S. 1999, A\&A, 350, 791

Jarrett, T. H., Chester, T., Cutri, R., Schneider, S. E., \& Huchra, J. P. 2003, AJ, 125, 525 
Kamphuis, J., \& Briggs, F. 1992, A\&A, 253, 335

Kennicutt, R. C., \& Hodge, P. W. 1980, ApJ, 241, 573

Kennicutt, R. C., Jr., et al. 2003, PASP, 115, 928

Kippenhahn, R., \& Weigert, A. 1990, Stellar Structure and Evolution, XVI, 468 pp. 192 figs.. Springer-Verlag Berlin Heidelberg New York. Also Astronomy and Astrophysics Library

Knapen, J. H., Stedman, S., Bramich, D. M., Folkes, S. L., \& Bradley, T. R. 2004, A\&A, 426, 1135

Kochanek, C. S., et al. 2001, ApJ, 560, 566

Kong, X., et al. 2000, AJ, 119, 2745

Kormendy, J. 1977, ApJ, 217, 406

Kormendy, J. 1993, Galactic Bulges, 153, 209

Kormendy, J., \& Kennicutt, R. C., Jr. 2004, ARA\&A, 42, 603

Kotulla, R., Fritze, U., Weilbacher, P., \& Anders, P. 2009, MNRAS, 396, 462

Kuntz, K. D., Harrus, I., McGlynn, T. A., Mushotzky, R. F., \& Snowden, S. L. 2008, PASP, 120, 740

Kurucz, R. L. 1992, The Stellar Populations of Galaxies, 149, 225

La Vigne, M. A., Vogel, S. N., \& Ostriker, E. C. 2006, ApJ, 650, 818

Le Borgne, J.-F., et al. 2003, A\&A, 402, 433

Leitherer, C., et al. 1999, ApJS, 123, 3

Lejeune, T., Cuisinier, F., \& Buser, R. 1997, A\&AS, 125, 229

Li, J.-L., Zhou, X., Ma, J., \& Chen, J.-S. 2004, Chinese Journal of Astronomy and Astrophysics, 4, 143

Lin, D. N. C., \& Pringle, J. E. 1987, ApJ, 320, L87

Long, K. S., Charles, P. A., \& Dubus, G. 2002, ApJ, 569, 204

Lynden-Bell, D. 1979, MNRAS, 187, 101 
Ma, J., Fan, Z., de Grijs, R., Wu, Z., Zhou, X., Wu, J., Jiang, Z., \& Chen, J. 2009, AJ, 137, 4884

Ma, J., et al. 2009, Research in Astronomy and Astrophysics, 9, 641

Maeder, A., \& Meynet, G. 1989, A\&A, 210, 155

Martin, D. C., et al. 2005, ApJ, 619, L1

McCarthy, D. W., Jr., Ge, J., Hinz, J. L., Finn, R. A., \& de Jong, R. S. 2001, PASP, 113, 353

McDonald, M., Courteau, S., \& Tully, R. B. 2009, MNRAS, 393, 628

Morrissey, P., et al. 2007, ApJS, 173, 682

Natali, G., Pedichini, F., \& Righini, M. 1992, A\&A, 256, 79

Noguchi, M. 1988, A\&A, 203, 259

Oke, J. B., \& Gunn, J. E. 1983, ApJ, 266, 713

Papovich, C., Dickinson, M., \& Ferguson, H. C. 2001, ApJ, 559, 620

Pickles, A. J. 1998, PASP, 110, 863

Regan, M. W., Thornley, M. D., Helfer, T. T., Sheth, K., Wong, T., Vogel, S. N., Blitz, L., \& Bock, D. C.-J. 2001, ApJ, 561, 218

Regan, M. W., \& Teuben, P. 2003, ApJ, 582, 723

Regan, M. W., et al. 2006, ApJ, 652, 1112

Renzini, A., \& Voli, M. 1981, A\&A, 94, 175

Salpeter, E. E. 1955, ApJ, 121, 161

Sánchez, S. F., Rosales-Ortega, F. F., Kennicutt, R. C., Johnson, B. D., Diaz, A. I., Pasquali, A., \& Hao, C. N. 2011, MNRAS, 410, 313

Schaller, G., Schaerer, D., Meynet, G., \& Maeder, A. 1992, A\&AS, 96, 269

Schlegel, D. J., Finkbeiner, D. P., \& Davis, M. 1998, ApJ, 500, 525

Schoenberner, D. 1983, ApJ, 272, 708 
Seigar, M. S. 2002, A\&A, 393, 499

Sérsic, J. L. 1968, Cordoba, Argentina: Observatorio Astronomico, 1968

Shostak, G. S., \& van der Kruit, P. C. 1984, A\&A, 132, 20

Skrutskie, M. F., et al. 2006, AJ, 131, 1163

Stetson, P. B. 1987, PASP, 99, 191

Talent, D. L. 1983, PASP, 95, 986

Tremaine S. 1989. In Dynamics of Astrophysical Disks, ed. JA Sellwood, p. 231. Cam- bridge: Cambridge Univ. Press

Toomre, A., \& Toomre, J. 1972, ApJ, 178, 623

Vassiliadis, E., \& Wood, P. R. 1993, ApJ, 413, 641

Wakker, B. P., \& Adler, D. S. 1995, AJ, 109, 134

Werner, M. W., et al. 2004, ApJS, 154, 1

Zhou, X., Jiang, Z.-J., Xue, S.-J., Wu, H., Ma, J., \& Chen, J.-S. 2001, Chinese Journal of Astronomy and Astrophysics, 1, 372 
Table 1: Filter information in the BATC photometric system and statistics of observations

\begin{tabular}{cccccccc}
\hline \hline No. & Filter & $\lambda_{\text {eff }^{\mathrm{a}}(\AA)}(\AA)$ & Bandwidth $(\AA)$ & $\operatorname{Exp}^{\mathrm{b}}(\mathrm{s})$ & $\mathrm{FWHM}^{\mathrm{c}}\left(^{\prime \prime}\right)$ & $\mathrm{N}^{\mathrm{d}}$ & $\mathrm{rms}^{\mathrm{e}}$ \\
\hline 01 & $a$ & 3360 & 222 & 13200 & 4.61 & 2 & 0.041 \\
02 & $b$ & 3890 & 187 & 8400 & 4.45 & 3 & 0.016 \\
03 & $c$ & 4210 & 185 & 7200 & 4.01 & 6 & 0.008 \\
04 & $d$ & 4550 & 222 & 11400 & 3.43 & 5 & 0.015 \\
05 & $e$ & 4920 & 225 & 13800 & 3.73 & 3 & 0.009 \\
06 & $f$ & 5270 & 211 & 11100 & 4.04 & 3 & 0.006 \\
07 & $g$ & 5795 & 176 & 6000 & 4.44 & 2 & 0.002 \\
08 & $h$ & 6075 & 190 & 6000 & 3.48 & 4 & 0.004 \\
09 & $i$ & 6660 & 312 & 3900 & 4.28 & 5 & 0.005 \\
10 & $j$ & 7050 & 121 & 6600 & 4.24 & 7 & 0.005 \\
11 & $k$ & 7490 & 125 & 7500 & 5.07 & 5 & 0.009 \\
12 & $m$ & 8020 & 179 & 7800 & 5.25 & 1 & 0.021 \\
13 & $n$ & 8480 & 152 & 10800 & 4.44 & 5 & 0.005 \\
14 & $o$ & 9190 & 194 & 15300 & 4.57 & 8 & 0.009 \\
15 & $p$ & 9745 & 188 & 19900 & 4.89 & 1 & 0.020 \\
\hline
\end{tabular}

${ }^{a}$ Effective wavelength of each filter

${ }^{b}$ Total exposure time of the combined image

${ }^{b}$ Full width at half maximum (FWHM) of the combined image

${ }^{d}$ Image number for calibration

${ }^{e}$ Calibration error in magnitude as explained in Section 3.1 


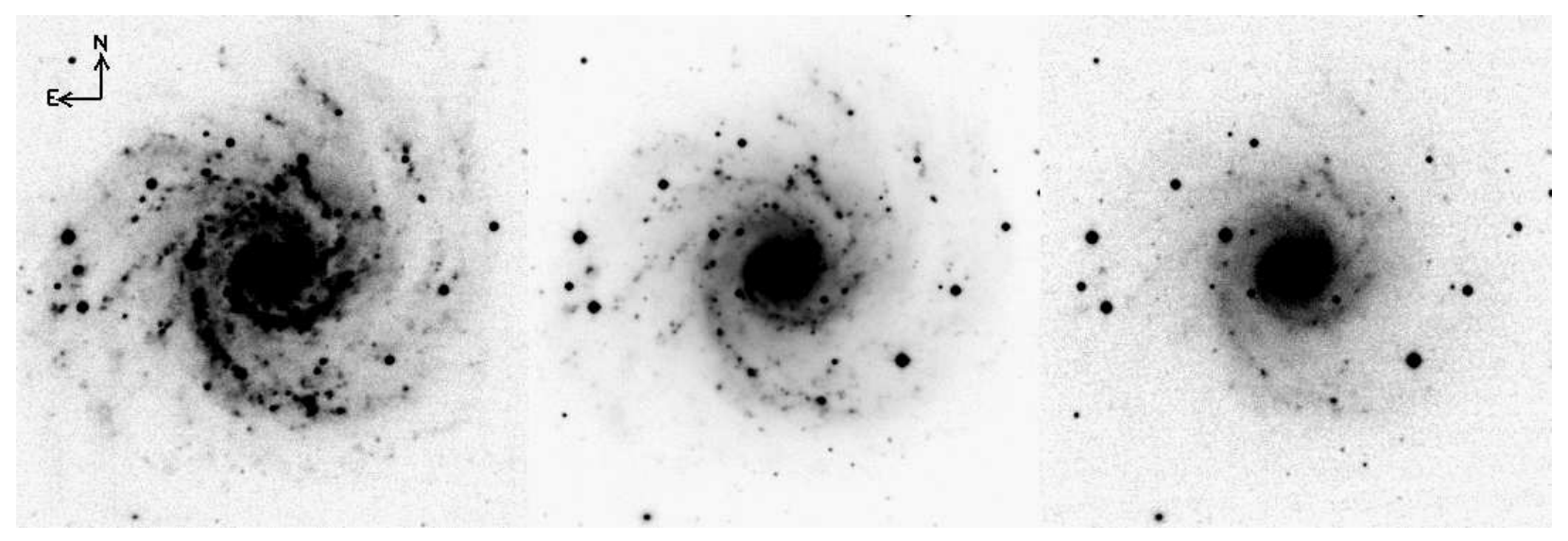

Fig. 1. - Images of NGC 628 in three BATC bands centered at the wavelengths of 3890 (left), 6660 (middle), and $9190 \AA$ (right). The image scale is $361 \times 361\left(1.71^{\prime \prime} /\right.$ pixel). 
Table 2: Multi-band observations from different telescopes for NGC 628

\begin{tabular}{|c|c|c|c|c|c|c|c|c|}
\hline $\mathrm{Name}^{\mathrm{a}}$ & Filter ${ }^{b}$ & $\begin{array}{l}\lambda_{\text {eff }^{\mathrm{c}}} \\
(\AA)\end{array}$ & $\begin{array}{c}\text { Bandwidth } \\
(\AA)\end{array}$ & $\mathrm{FOV}^{\mathrm{d}}$ & $\begin{array}{c}\text { scale }^{\mathrm{e}} \\
\left({ }^{\prime \prime}\right)\end{array}$ & $\begin{array}{l}\operatorname{Exp}^{f} \\
(\mathrm{~s})\end{array}$ & $\begin{array}{c}\text { FWHMg } \\
\left({ }^{\prime \prime}\right)\end{array}$ & Reference \\
\hline \multirow{2}{*}{ GALEX } & FUV & 1516 & 268 & $1.28^{\circ}$ & 1.5 & 1636.05 & 4.3 & \multirow[b]{2}{*}{1} \\
\hline & NUV & 2267 & 732 & $1.24^{\circ}$ & 1.5 & 1636.05 & 5.3 & \\
\hline \multirow{3}{*}{ XMM-OM } & UVW1 & 2905 & 620 & $17^{\prime}$ & 0.95 & $\sim 1000.0$ & 2.0 & \multirow{3}{*}{2} \\
\hline & UVM2 & 2298 & 439 & $17^{\prime}$ & 0.95 & $\sim 1000.0$ & 1.8 & \\
\hline & UVW2 & 2070 & 500 & $17^{\prime}$ & 0.95 & $\sim 1000.0$ & 2.0 & \\
\hline BATC & $a-p$ & $3000-9900$ & $120-310$ & $58^{\prime}$ & 1.7 & $1.1-5 \mathrm{~h}$ & $\sim 4.3$ & 3 \\
\hline \multirow{3}{*}{ 2MASS } & $J$ & $1.235 \mu \mathrm{m}$ & $0.162 \mu \mathrm{m}$ & $0.39^{\circ}$ & 1 & 7.8 & $\sim 2.5$ & \multirow{3}{*}{4} \\
\hline & $H$ & $1.662 \mu \mathrm{m}$ & $0.251 \mu \mathrm{m}$ & $0.39^{\circ}$ & 1 & 7.8 & $\sim 2.5$ & \\
\hline & $K_{s}$ & $2.159 \mu \mathrm{m}$ & $0.262 \mu \mathrm{m}$ & $0.39^{\circ}$ & 1 & 7.8 & $\sim 2.5$ & \\
\hline Bok & $K_{s}$ & $2.16 \mu \mathrm{m}$ & $0.262 \mu \mathrm{m}$ & $8.5^{\prime}$ & 0.24 & $\sim 1500$ & 1.5 & 5 \\
\hline \multirow{4}{*}{ Spitzer } & IRAC1 & $3.550 \mu \mathrm{m}$ & $0.75 \mu \mathrm{m}$ & $5.2^{\prime}$ & 0.75 & 1500.8 & 1.66 & \multirow{4}{*}{6} \\
\hline & IRAC2 & $4.493 \mu \mathrm{m}$ & $1.01 \mu \mathrm{m}$ & $5.2^{\prime}$ & 0.75 & 1500.8 & 1.72 & \\
\hline & IRAC3 & $5.731 \mu \mathrm{m}$ & $1.42 \mu \mathrm{m}$ & $5.2^{\prime}$ & 0.75 & 1500.8 & 1.88 & \\
\hline & IRAC4 & $7.872 \mu \mathrm{m}$ & $2.93 \mu \mathrm{m}$ & $5.2^{\prime}$ & 0.75 & 1500.8 & 1.98 & \\
\hline
\end{tabular}

${ }^{a}$ Name of the telescope or survey

${ }^{b}$ Name of the filter

${ }^{c}$ Effective wavelength in angstrom if not specified

${ }^{d}$ Field of view of the CCD camera

${ }^{e}$ Mosaic pixel scale

${ }^{f}$ Total exposure time in unit of seconds if not specified

${ }^{g}$ Full width at half maximum of the mosaic

Note. - References. (1) Morrissev et al. (2007); (2) Kuntz et al. (2008); (3) Zhou et al. (2001); (4) Skrutskie et al. (2006); (5) Knapen et al. (2004); (6) Fazio et al. (2004) 


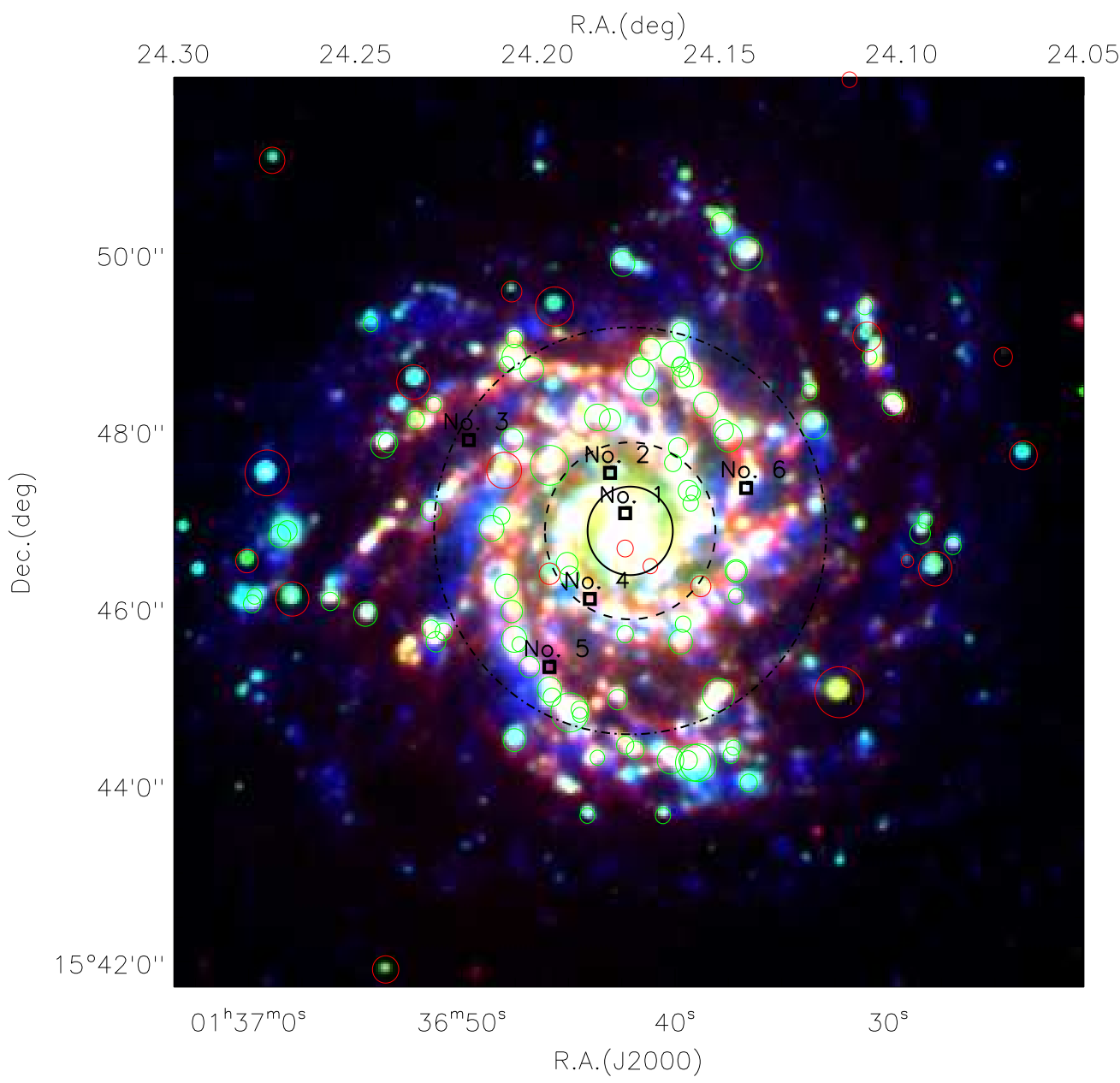

Fig. 2.- True color map of NGC 628 synthesized with the $8 \mu \mathrm{m}$ band (red), H $\alpha$ narrow band (6563 $\AA$, green) and NUV band (2271 $\AA$, blue) images. These bands are chosen to present the young stellar population and dust emission. Red and green solid circles are stars and HII regions to be removed. Only those regions with radius larger than 5 arcsec in the catalogue of Fathi et al. (2007) are displayed in this figure. Black squares are the selected pixels for illustrating the model fitting goodness in section 4.3. The solid circle around the center, the dashed circle, and the outmost dash-dotted circle represent the bulge together with the transition zone within $R<0.5^{\prime}$, the outer boundary of the inner region of the disk at 1 arcmin, and the outer boundary of the outer disk at about $2.2^{\prime}$ where the valid SEDs are still available (see Section 5.3), respectively. 

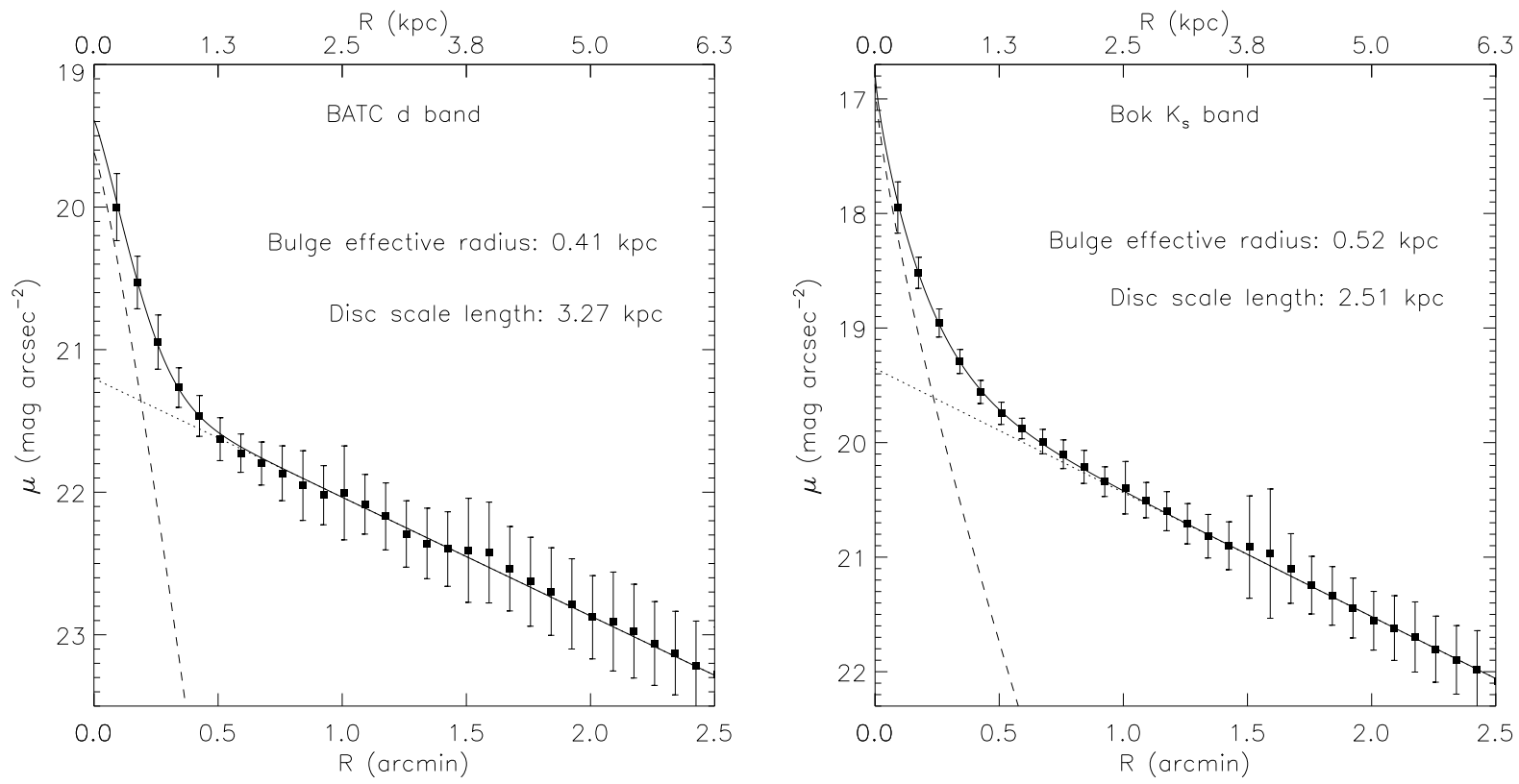

Fig. 3.- Left: radial surface brightness profile of the BATC $d$ band as plotted in filled squares with error bars. Right: radial surface brightness profile of the BATC $K_{s}$ band as plotted in filled squares with error bars. The dashed curve and dotted line in both panels are the fitted Sérsid (1968) law for the bulge and exponential law for the disk, respectively. The solid curve is the sum of these two fitted components. 
Table 3: Observed SEDs of several sampled pixels and corresponding fitted parameters

\begin{tabular}{|c|c|c|c|c|c|c|c|c|c|c|c|c|c|c|c|c|c|c|c|c|c|c|c|c|c|c|c|c|c|c|c|}
\hline No & $\mathrm{X}$ & $\mathrm{Y}$ & $\alpha(\mathrm{J} 2000)$ & $\delta(\mathrm{J} 2000)$ & NUV & UVM2 & UVW1 & $a$ & $b$ & $c$ & $d$ & $e$ & $f$ & $g$ & $h$ & $i$ & $j$ & $k$ & $m$ & $n$ & $o$ & $p$ & $J$ & $H$ & $K_{s} 1$ & IRAC1 & IRAC2 & Age $\mathrm{E}$ & $\mathrm{E}(\mathrm{B}-\mathrm{V})$ & $\mathrm{Z}$ & {$[\mathrm{Fe} / \mathrm{H}]$} \\
\hline 1 & 182 & 189 & 24.1739 & 15.7868 & 22.76 & 22.89 & 21.67 & 20.92 & $\overline{20.191}$ & 19.78 & 19.46 & 19.15 & $\overline{18.911}$ & 18.651 & $\overline{18.611}$ & 18.42 & 18.30 & 18.12 & 18.05 & 17.98 & 17.82 & 17.73 & 17.54 & 17.33 & 17.52 & 18.32 & 18.83 & 13.2 & 0.34 & 0.0012 & -1.18 \\
\hline$\cdots$ & $\ldots$ & & & & 0.18 & 0.18 & 0.12 & 0.09 & 0.06 & 0.05 & 0.05 & 0.04 & 0.03 & 0.03 & 0.03 & 0.03 & 0.03 & 0.02 & 0.03 & 0.03 & 0.03 & 0.03 & 0.02 & 0.02 & 0.03 & 0.03 & 0.03 & 0.6 & 0.01 & 0.0001 & 0.04 \\
\hline 2 & 188 & 205 & 24.1766 & 15.7945 & 23.48 & 23.90 & 22.65 & 21.91 & 21.32 & 20.98 & 20.66 & 20.38 & 20.131 & 19.941 & 19.901 & 19.72 & 19.62 & 19.48 & 19.40 & 19.36 & 19.20 & 19.14 & 19.09 & 18.68 & 18.95 & 19.73 & 20.24 & 8.5 & 0.30 & 0.0016 & -1.09 \\
\hline & & & & & 0.27 & 0.37 & 0.19 & 0.15 & 0.11 & 0.09 & 0.08 & 0.07 & 0.06 & 0.06 & 0.06 & 0.05 & 0.05 & 0.05 & 0.07 & 0.06 & 0.07 & 0.07 & 0.06 & 0.06 & 0.08 & 0.05 & 0.06 & 0.9 & 0.01 & 0.0003 & 0.08 \\
\hline 3 & 244 & 218 & 24.2041 & 15.8014 & 24.84 & 99.99 & 23.65 & 23.06 & 22.412 & 22.28 & 21.96 & 21.79 & 21.522 & 21.342 & 21.372 & 21.25 & 21.16 & 21.04 & 20.89 & 20.73 & 20.61 & 20.70 & 20.28 & $20.31 \mathrm{~s}$ & 99.99 & & 21.66 & 1.1 & & 0.0010 & -1.23 \\
\hline & & & & & 0.44 & 9.99 & 0.30 & 0.33 & 0.20 & 0.21 & 0.17 & 0.14 & 0.12 & 0.11 & 0.12 & 0.12 & 0.12 & 0.12 & 0.28 & 0.16 & 0.18 & 0.29 & 0.17 & 0.26 & 9.99 & 0.10 & 0.12 & 0.3 & 0.03 & 0.0005 & 0.20 \\
\hline 4 & 196 & 155 & 24.1813 & 15.7708 & 23.52 & 23.86 & 22.65 & 21.98 & 21.32 & 21.04 & 20.74 & 20.49 & 20.27 & 20.082 & 20.05 & 19.86 & 19.77 & 19.64 & 19.57 & 19.49 & 19.35 & 19.27 & 19.28 & 18.92 & 19.07 & 19.83 & 20.29 & 3.2 & 0.37 & 0.0016 & -1.04 \\
\hline & & & & & 0.28 & 0.28 & 0.18 & 0.16 & 0.11 & 0.09 & 0.08 & 0.07 & 0.07 & 0.06 & 0.06 & 0.06 & 0.05 & 0.05 & 0.08 & 0.07 & 0.07 & 0.08 & 0.08 & 0.07 & 0.09 & 0.05 & 0.06 & 0.4 & 0.01 & 0.0003 & 0.09 \\
\hline 5 & 212 & 128 & 24.1895 & 15.7582 & 23.82 & 23.62 & 23.07 & 22.56 & 21.87 & 21.60 & 21.45 & 21.18 & 20.97 & 20.862 & 20.82 & 20.67 & 20.60 & 20.49 & 20.43 & 20.36 & 20.30 & 20.22 & 19.98 & 20.012 & 20.08 & 20.76 & 21.24 & 2.6 & 0.27 & 0.0032 & -0.74 \\
\hline & & & & & 0.29 & 0.31 & 0.22 & 0.23 & 0.15 & 0.13 & 0.12 & 0.10 & 0.09 & 0.09 & 0.09 & 0.08 & 0.09 & 0.09 & 0.15 & 0.13 & 0.16 & 0.17 & 0.12 & 0.14 & 0.19 & 0.08 & 0.10 & 0.5 & 0.02 & 0.0010 & 0.19 \\
\hline 6 & 134 & 199 & 24.1501 & 15.7909 & 22.37 & 22.60 & 21.81 & 21.51 & 21.072 & 20.98 & 20.80 & 20.64 & 20.49 & 20.392 & 20.39 & 20.20 & 20.19 & 20.07 & 20.05 & 19.91 & 19.77 & 19.81 & 19.43 & 19.51 & 19.58 & 20.16 & 20.59 & 1.3 & 0.18 & 0.0302 & 0.30 \\
\hline & & & & & 0.16 & 0.18 & 0.12 & 0.11 & 0.09 & 0.09 & 0.08 & 0.08 & 0.07 & 0.07 & 0.07 & 0.07 & 0.07 & 0.07 & 0.11 & 0.09 & 0.10 & 0.13 & 0.08 & 0.10 & 0.14 & 0.06 & 0.08 & 0.2 & 0.02 & 0.0040 & 0.06 \\
\hline
\end{tabular}

$\mathrm{X}$ and $\mathrm{Y}$ are the pixel position in the image with the size of $361 \times 361$. The positions of these pixels are marked in Figure 1 Both right ascension $\alpha$ and declination $\delta$ are in degrees. The last four columns are the fitted parameters. There are two rows for each pixel: one row contains the observed SEDs and fitted results and the other includes their errors. Note that magnitudes of 99.99 and errors of 9.99 are set for the bands whose SNRs are three times lower than the sky background standard deviation. 

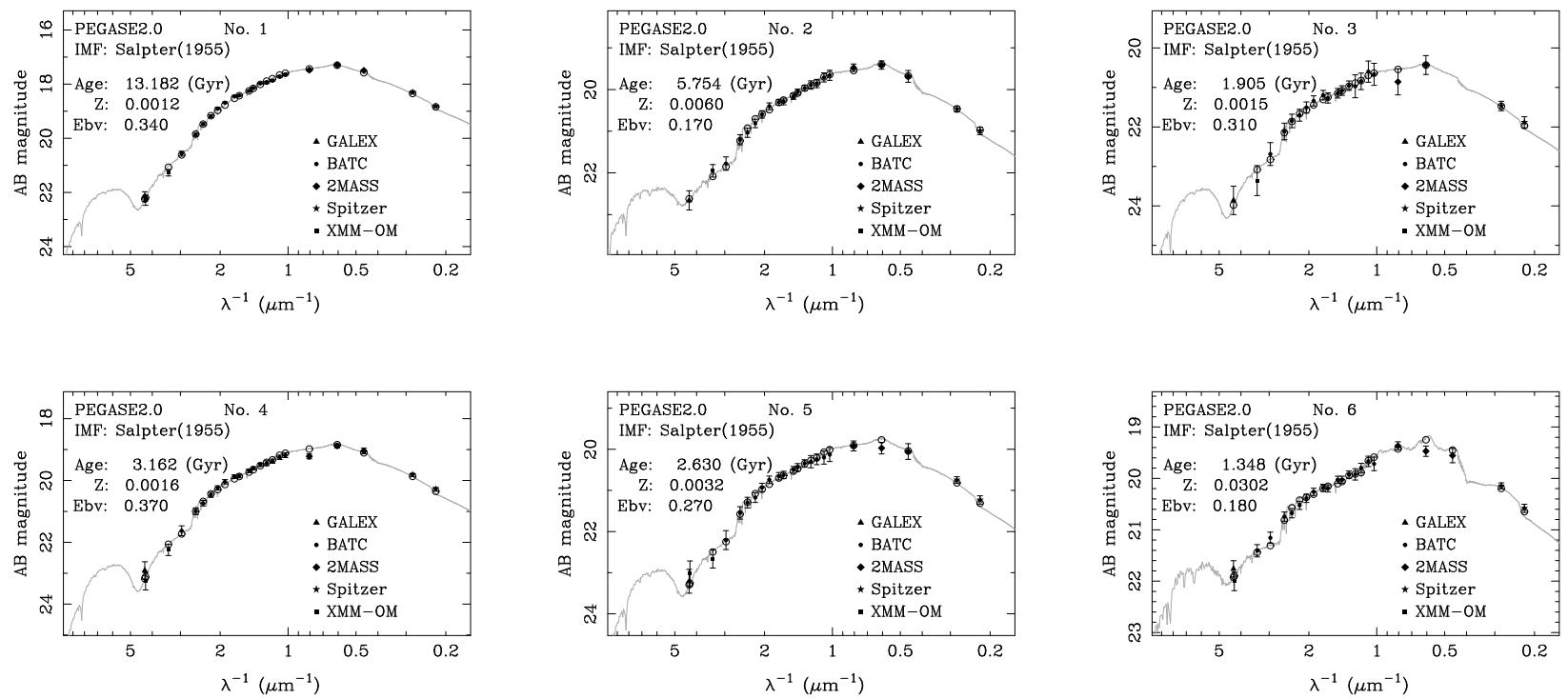

Fig. 4.- Combined plots of observed SEDs, matched model SEDs and corresponding spectra for six randomly sampled pixels as described in Table 3. The filled symbols are the observed AB magnitudes of specified bands and open circles are the matched model magnitudes. Spectra are drawn in grey. Vertical bars are the measured errors of the observed magnitudes as defined in Equation 2 , 

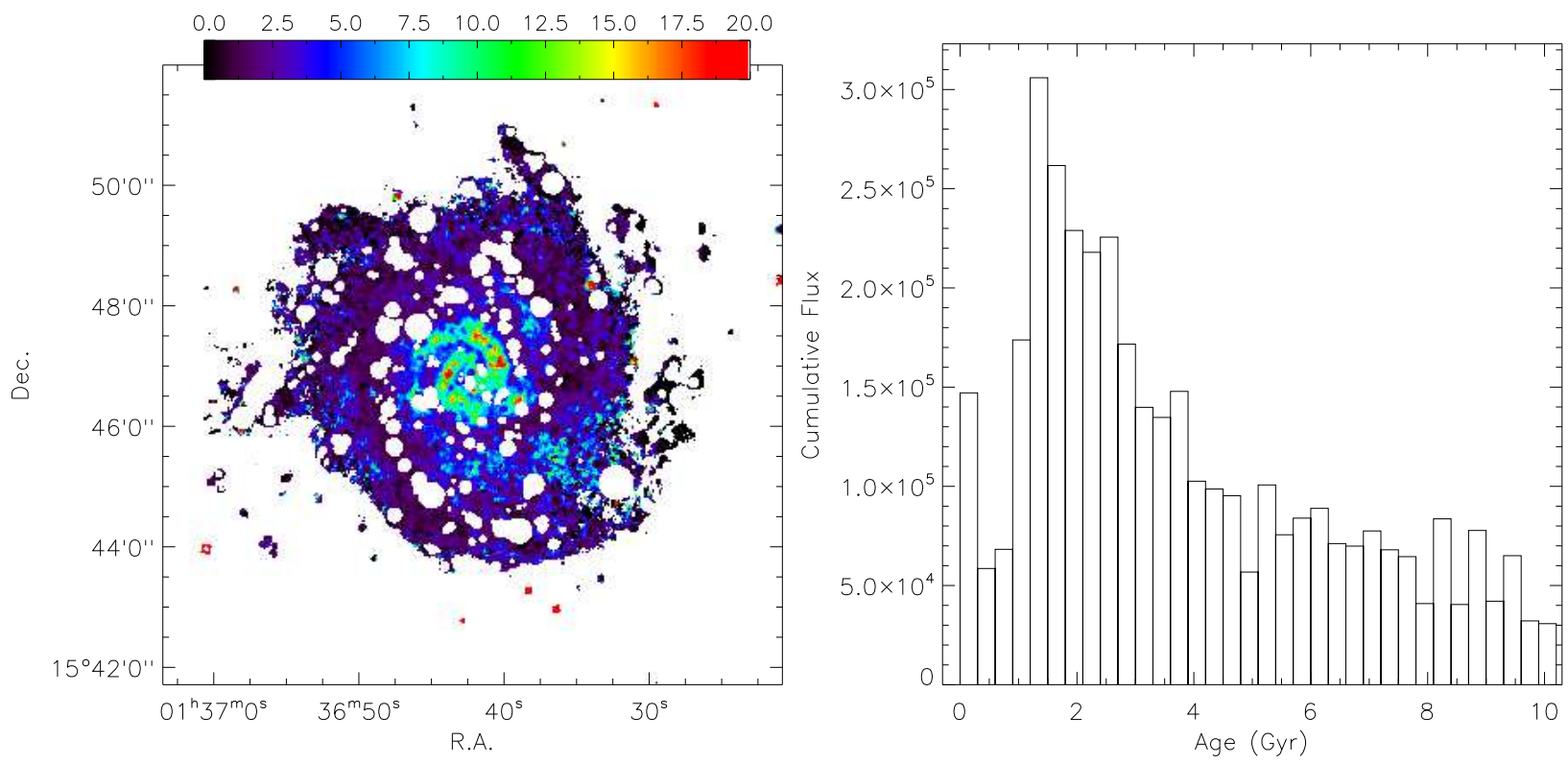

Fig. 5.- Left: two-dimensional age distribution of NGC 628. Blank regions are masked stars and HiI regions. Right: histogram distribution of mass weighted ages. The cumulative flux in the vertical ordinate is the total apparent luminosity of the $K_{s}$ bands in $10^{-30} \mathrm{ergs} \mathrm{s}^{-1} \mathrm{~cm}^{-2} \mathrm{~Hz}^{-1} \operatorname{arcsec}^{-2}$ which represents the stellar mass. 

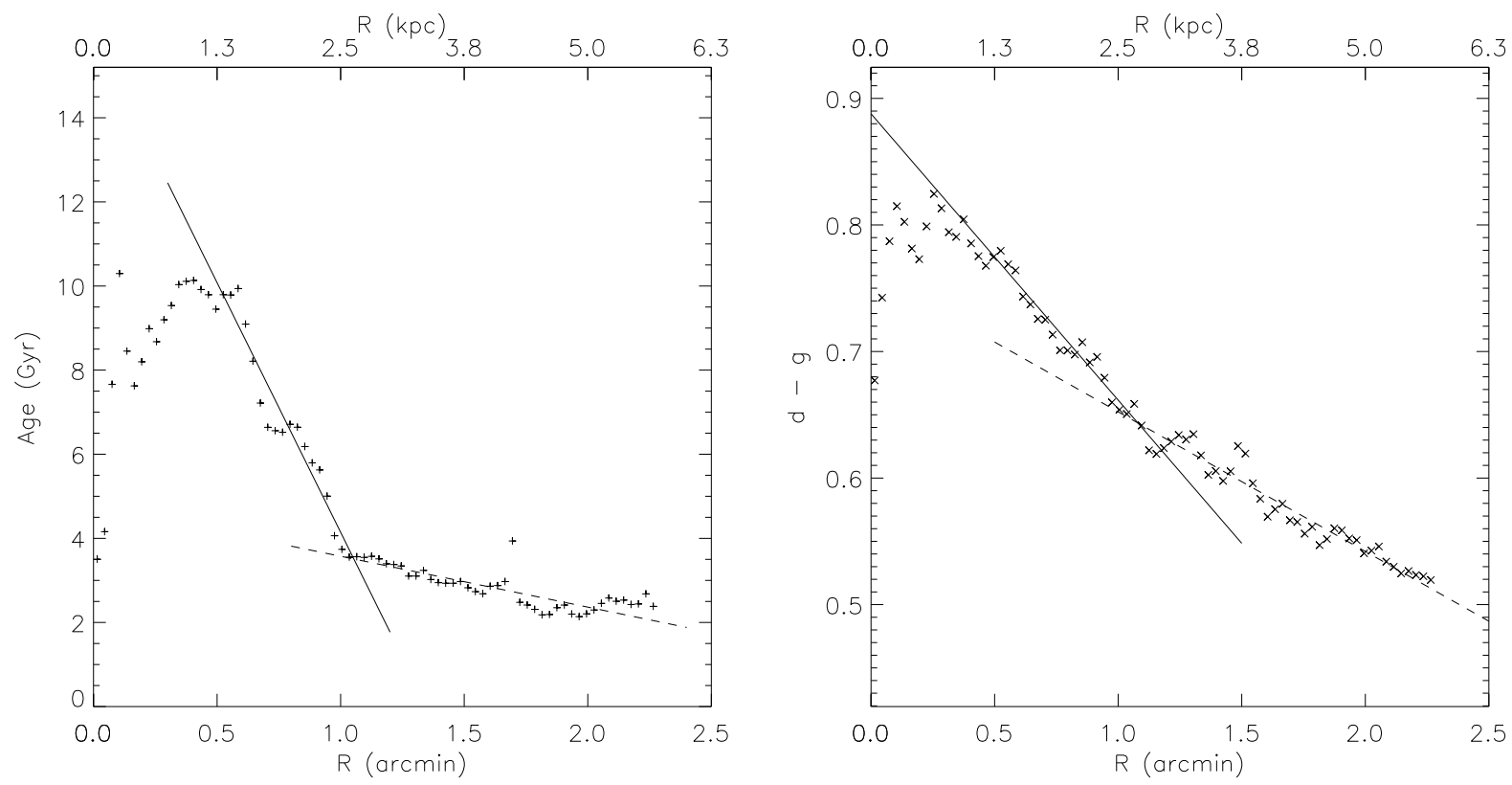

Fig. 6. - Left: radial age profile. Right: radial profile of the BATC $d-g$ color. The solid and dashed lines present two fitted gradients in the inner region of the disk $\left(0.5-1.0^{\prime}\right)$ and the outer region of the disk $\left(1.0-2.2^{\prime}\right)$, respectively. 

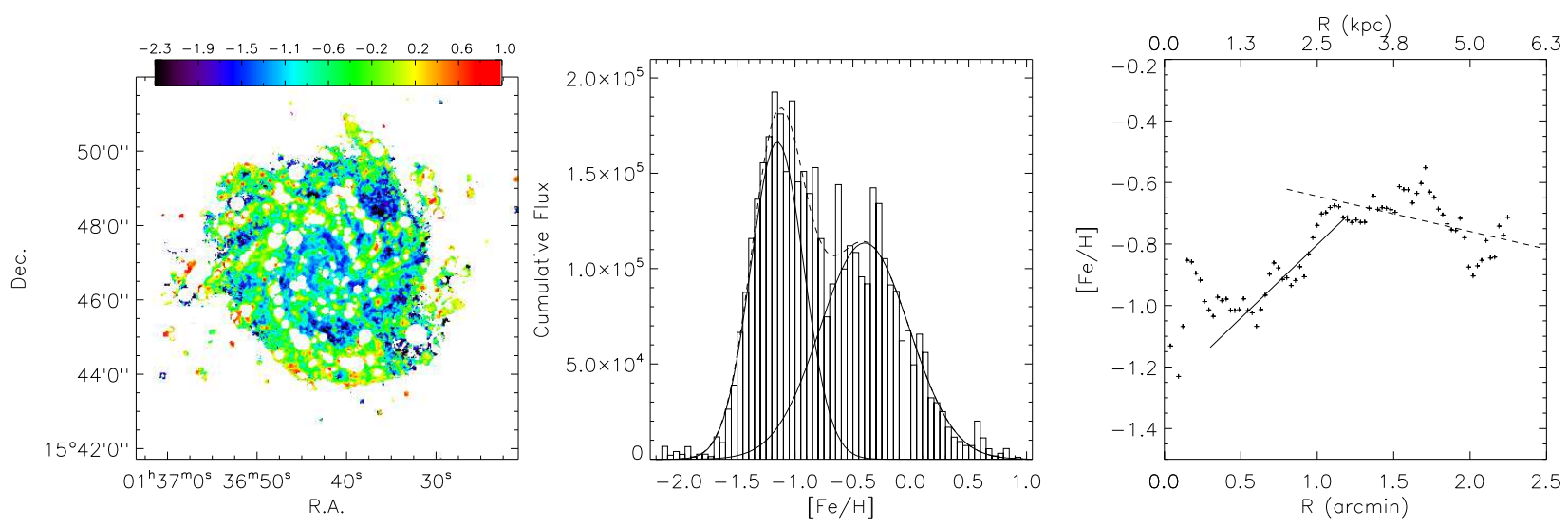

Fig. 7.- Left: two-dimensional $[\mathrm{Fe} / \mathrm{H}]$ distribution of NGC 628. Blank regions are masked stars and HiI regions. Middle: histogram distribution of mass weighted metallicity. The cumulative flux in the vertical ordinate is the total apparent luminosity of the $K_{s}$ bands in $10^{-30} \mathrm{ergs} \mathrm{s}^{-1} \mathrm{~cm}^{-2} \mathrm{~Hz}^{-1} \operatorname{arcsec}^{-2}$. Two components of poor and rich metallicity are fitted by a double Gaussian function (bimodal), which is plotted in the dashed curve. The solid curves are two single Gaussian items of the bimodal. Right: radial $[\mathrm{Fe} / \mathrm{H}]$ distribution. The solid line and dashed lines show the $[\mathrm{Fe} / \mathrm{H}]$ gradients within the range of $0.5-1.0^{\prime}$ and the range of $1.0-2.5^{\prime}$, respectively. 
Table 4: Bimodal parameters of the $[\mathrm{Fe} / \mathrm{H}]$ distribution fitted by a double Gaussian function

\begin{tabular}{c|c|c|c|c|c|c}
\hline \hline Abundance & $\mu^{\mathrm{a}}$ & Error $_{\mu}^{\mathrm{b}}$ & $\sigma^{\mathrm{c}}$ & Error $_{\sigma}$ & $A^{\mathrm{d}}$ & Error $_{A}$ \\
\hline poor & -1.15 & 0.03 & 0.23 & 0.03 & $1.66 \times 10^{5}$ & $0.20 \times 10^{5}$ \\
\hline rich & -0.40 & 0.08 & 0.39 & 0.07 & $1.13 \times 10^{5}$ & $0.09 \times 10^{5}$ \\
\hline
\end{tabular}

${ }^{a}$ Mathematical expectation of Gaussian function

${ }^{b}$ Error (for all parameters) with $95 \%$ confidence

${ }^{c}$ Standard deviation of Gaussian function

${ }^{d}$ Constant coefficient of Gaussian function 

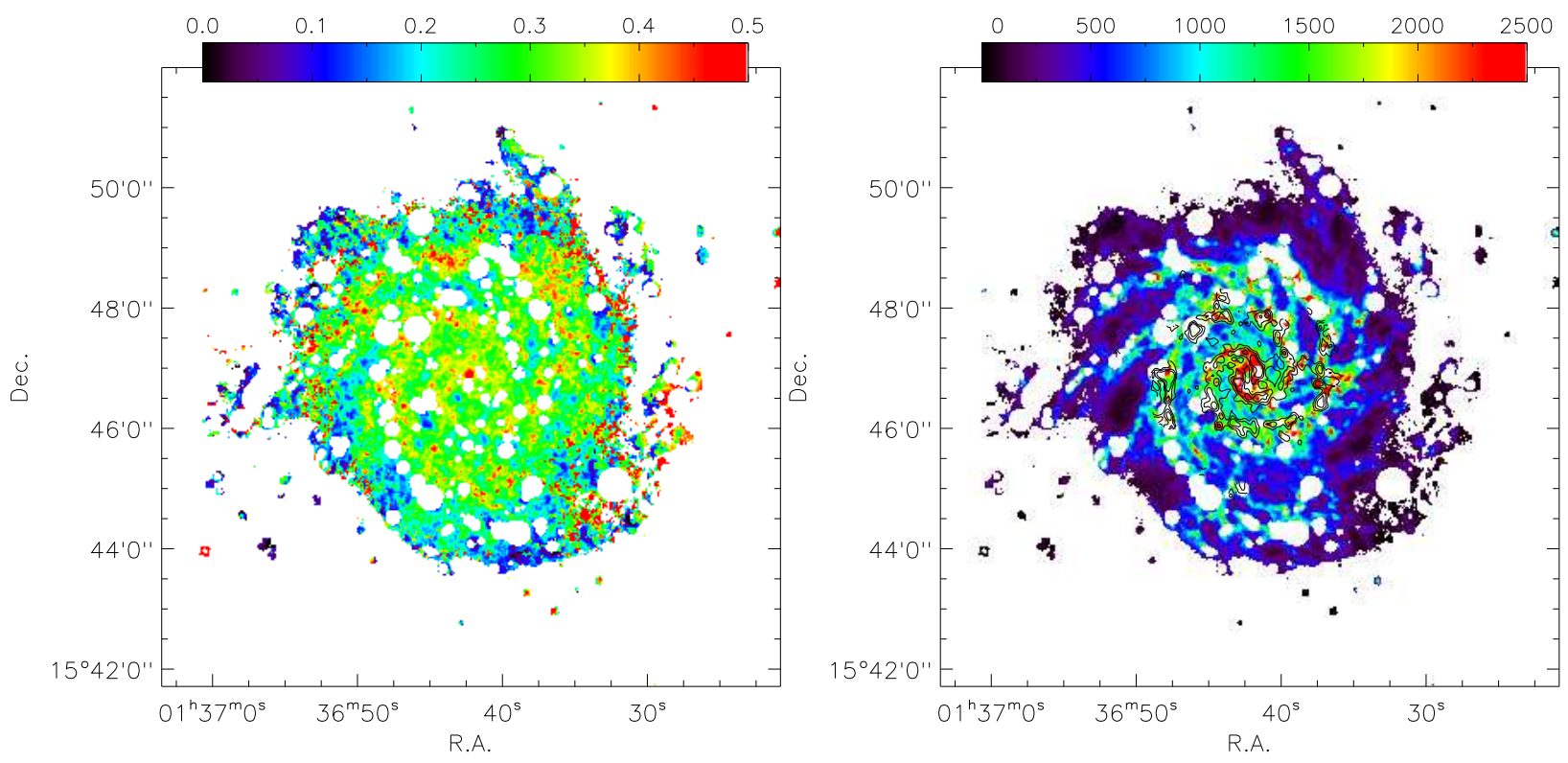

Fig. 8.- Left: two-dimensional intrinsic reddening distribution of NGC 628. Blank regions are masked stars and HII regions. Right: IRAC $8.0 \mu \mathrm{m}$ stellar flux-subtracted image in the same area as the reddening map. Overlapped contours show CO intensities (contour levels: $1.5,3$, and $\left.5 \mathrm{Jybeam}^{-1} \mathrm{kms}^{-1}\right)$. 

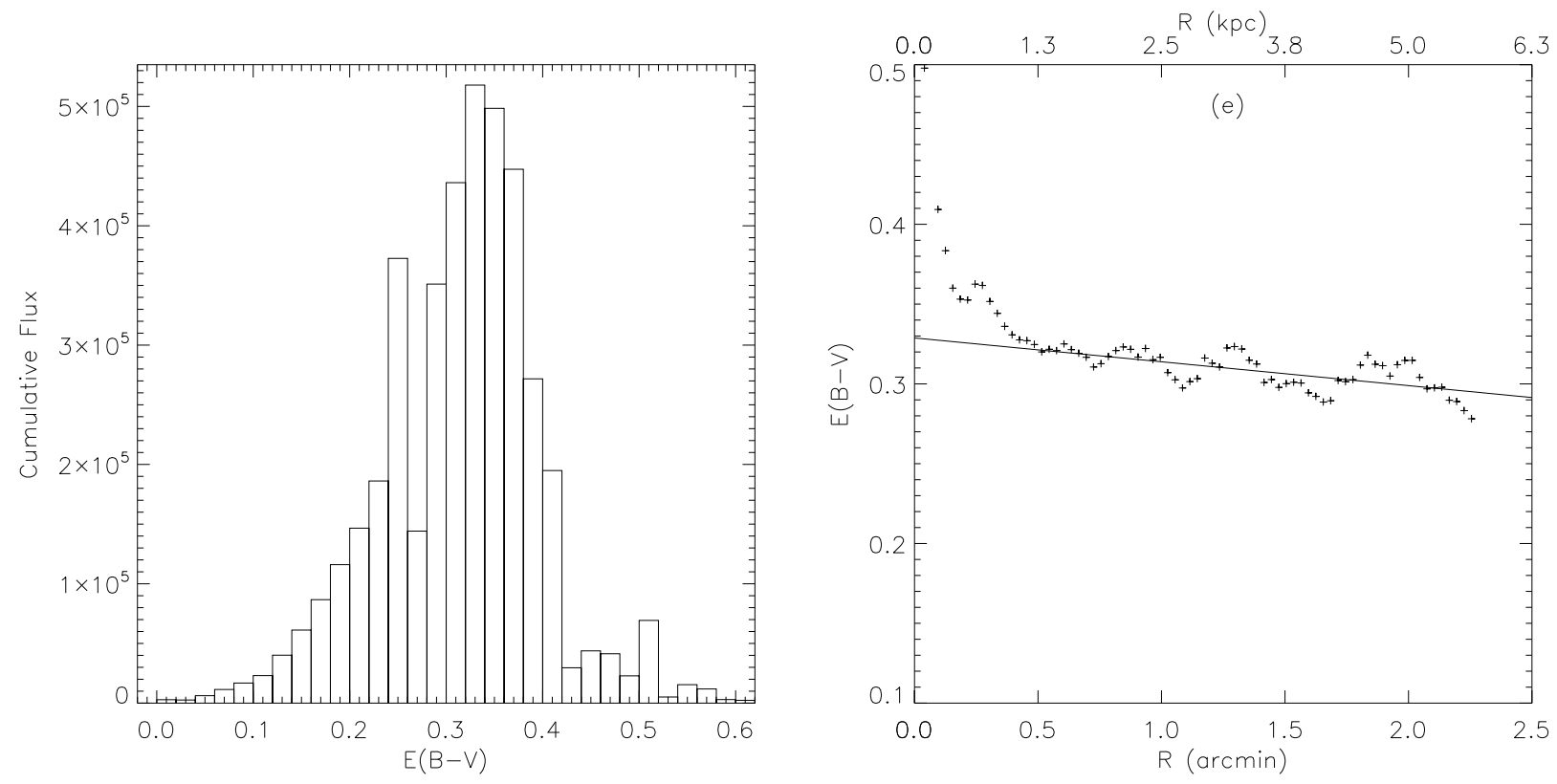

Fig. 9.- Left: histogram distribution of the overall intrinsic reddening in $E(B-V)$. The cumulative flux in the vertical ordinate is the total apparent luminosity of the $K_{s}$ bands in $10^{-30} \mathrm{ergs} \mathrm{s}^{-1} \mathrm{~cm}^{-2} \mathrm{~Hz}^{-1} \operatorname{arcsec}^{-2}$. Right: radial distribution of the intrinsic reddening. The solid line presents the fitted radial gradient. 